\title{
Supplementary Material to Graph connection Laplacian methods can be made robust to noise
}

\author{
By Noureddine El Karoui ${ }^{*, \ddagger}$ HaU-Tieng Wu $\mathrm{U}^{\dagger, \S}$ \\ UC Berkeley ${ }^{\ddagger}$ and University of Toronto ${ }^{\S}$
}

\section{APPENDIX A: PROOFS OF RESULTS IN THE MAIN PAPER}

\section{A-1. Proof of Theorem 2.1 in [21].}

Proof. We first note that $N_{i}^{\vee}-\mathrm{O} N_{j}^{\vee} \sim \mathcal{N}\left(0, \Sigma_{i}+\mathrm{O} \Sigma_{j} \mathrm{O}^{\prime}\right)$. Applying Lemma C-2 to $\left\|N_{i}^{\vee}-\mathrm{O} N_{j}^{\vee}\right\|^{2}$ with $Q_{i}=\mathrm{Id}$, we get

$$
\begin{aligned}
& \sup _{\mathrm{O} \in \mathcal{T}} \sup _{i \neq j}\left|\left\|N_{i}^{\vee}-\mathrm{O} N_{j}^{\vee}\right\|^{2}-\operatorname{trace}\left(\Sigma_{i}+\mathrm{O} \Sigma_{j} \mathrm{O}^{\prime}\right)\right|= \\
& \mathrm{O}_{P}\left(\sqrt{\log \left[\operatorname{Card}\{\mathcal{T}\} n^{2}\right]} \sup _{i, j, \mathrm{O}}\left[\sqrt{\operatorname{trace}\left(\left(\Sigma_{i}+\mathrm{O} \Sigma_{j} \mathrm{O}^{\prime}\right)^{2}\right)}\right]\right. \\
& \left.+\log \left[\operatorname{Card}\{\mathcal{T}\} n^{2}\right] \sup _{i, j, \mathrm{O}}\left\|\left(\Sigma_{i}+\mathrm{O} \Sigma_{j} \mathrm{O}^{\prime}\right)\right\|_{2}\right) .
\end{aligned}
$$

Of course, using the fact that for positive semi-definite matrices, $(A+B)^{2} \preceq$ $2\left(A^{2}+B^{2}\right)$ in the positive-semidefinite order, we have

$$
\operatorname{trace}\left(\left(\Sigma_{i}+\mathrm{O} \Sigma_{j} \mathrm{O}^{\prime}\right)^{2}\right) \leq 2 \operatorname{trace}\left(\Sigma_{i}^{2}+\left[\mathrm{O} \Sigma_{j} \mathrm{O}^{\prime}\right]^{2}\right) .
$$

Hence,

$$
\begin{aligned}
& \sup _{\mathrm{O} \in \mathcal{T}} \sup _{i \neq j}\left|\left\|N_{i}^{\vee}-\mathrm{O} N_{j}^{\vee}\right\|^{2}-\operatorname{trace}\left(\Sigma_{i}+\mathrm{O} \Sigma_{j} \mathrm{O}^{\prime}\right)\right|= \\
& \quad \mathrm{O}_{P}\left(\sqrt{\log \left[\operatorname{Card}\{\mathcal{T}\} n^{2}\right]} \sup _{i} \sup _{\mathrm{O} \in \mathcal{T}} \sqrt{\operatorname{trace}\left(\left[\mathrm{O} \Sigma_{i} \mathrm{O}^{\prime}\right]^{2}\right)}+\log \left[\operatorname{Card}\{\mathcal{T}\} n^{2}\right] \sup _{i} \sup _{\mathrm{O} \in \mathcal{T}}\left\|\mathrm{O} \Sigma_{i} \mathrm{O}^{\prime}\right\| \|_{2}\right) .
\end{aligned}
$$

We also note that

$$
\left(S_{i}^{\vee}-\mathrm{O} S_{j}^{\vee}\right)^{\prime}\left(N_{i}^{\vee}-\mathrm{O} N_{j}^{\vee}\right) \sim \mathcal{N}\left(0, \gamma_{i, j, \mathrm{O}}^{2}\right)
$$

*Support from NSF grant DMS-0847647 (CAREER) is gratefully acknowledged.

${ }^{\dagger}$ Support from AFOSR grant FA9550-09-1-0643 is gratefully acknowledged. The authors thank an anonymous referee for interesting and constructive comments that helped improve the paper.

AMS 2000 subject classifications: Primary 60F99; secondary 53A99

Keywords and phrases: concentration of measure, random matrices, Graph connection Laplacian, Vector diffusion maps, spectral geometry, kernel methods 
where

$$
\gamma_{i, j, \mathrm{O}}^{2}=\left(S_{i}^{\vee}-\mathrm{O} S_{j}^{\vee}\right)^{\prime}\left(\Sigma_{i}-\mathrm{O} \Sigma_{j} \mathrm{O}^{\prime}\right)\left(S_{i}^{\vee}-\mathrm{O} S_{j}^{\vee}\right)
$$

We note that

$\gamma_{i, j, \mathrm{O}}^{2} \leq 2\left(\left\|S_{i}^{\vee}\right\|^{2}+\left\|\mathrm{O} S_{j}^{\vee}\right\|^{2}\right)\left(\left\||| \Sigma_{i}\left|\left\|_{2}+\right\| \mathrm{OO} \Sigma_{j} \mathrm{O}^{\prime}\right|\right\|_{2}\right) \leq 8 \sup _{i, \mathrm{O} \in \mathcal{T}}\left\|\mathrm{O} \Sigma_{i} \mathrm{O}^{\prime}\right\|\left\|_{2} \sup _{i, \mathrm{O} \in \mathcal{T}}\right\| \mathrm{O} S_{i}^{\vee} \|^{2}$.

Recall also that it is well known that if $Z_{1}, \ldots, Z_{N}$ are $\mathcal{N}\left(0, \gamma_{i}^{2}\right)$ random variables,

$$
\sup _{1 \leq k \leq N}\left|Z_{k}\right|=\mathrm{O}_{P}\left(\sqrt{\log N} \sup _{k} \gamma_{k}\right) .
$$

This result can be obtained by a simple union bound argument. In our case, it means that

$$
\sup _{\mathrm{O} \in \mathcal{T}} \sup _{i \neq j}\left|\left(S_{i}^{\vee}-\mathrm{O} S_{j}^{\vee}\right)^{\prime}\left(N_{i}^{\vee}-\mathrm{O} N_{j}^{\vee}\right)\right|=\mathrm{O}_{P}\left(\sqrt{\log \left[\operatorname{Card}\{\mathcal{T}\} n^{2}\right]} \sup _{i, \mathrm{O} \in \mathcal{T}} \sqrt{\|\| \mathrm{O} \Sigma_{i} \mathrm{O}^{\prime}\|\|_{2}} \sup _{i, \mathrm{O} \in \mathcal{T}}\left\|\mathrm{O} S_{i}^{\vee}\right\|\right) .
$$

\section{A-2. Proof of Theorem 2.2.}

Proof. Clearly, $0_{i j, c l e a n}^{*}$ is a minimizer of $L_{i j}(0):=d_{i j, \text { clean }}^{2}(0)+\operatorname{trace}\left(\Sigma_{i}+\Sigma_{j}\right)$, since the second term does not depend on 0. Naturally, if Assumptions A0 and A1 apply to $d_{i j, \text { clean }}^{2}(0)$, they apply to $d_{i j, \text { clean }}^{2}(0)+C$, for $C$ any constant. In particular, taking $C=\operatorname{trace}\left(\Sigma_{i}+\Sigma_{j}\right)$, we see that Assumptions A0 and A1 apply to the function $L_{i j}(0)$.

The approximation results of Proposition 2.2 guarantee that, under Assumptions $\mathrm{G} 1, \mathrm{~A} 0$ and $\mathrm{A} 1, \mathrm{O}_{i j \text {,noisy }}^{*}$ is a near minimizer of $d_{i j \text {,clean }}^{2}(0)$. Indeed, we have by definition,

$$
d_{i j, \text { noisy }}^{2}\left(\mathrm{O}_{i j, \text { noisy }}^{*}\right) \leq d_{i j, \text { noisy }}^{2}\left(\mathrm{O}_{i j, \text { clean }}^{*}\right) .
$$

But under assumption G1, Proposition 2.2 and the fact that the elements of $\mathcal{T}_{\text {exact }}$ are orthogonal matrices imply that

$$
\forall 0 \in \mathcal{T}, \forall i \neq j d_{i j, \text { noisy }}^{2}(0)=L_{i j}(0)+\mathrm{O}_{P}\left(u_{n, p}\right) .
$$

Hence, we can rephrase Equation (A.1) as

$$
L_{i j}\left(\mathrm{O}_{i j, \text { noisy }}^{*}\right) \leq L_{i j}\left(\mathrm{O}_{i j, \text { clean }}^{*}\right)+\mathrm{O}_{P}\left(u_{n, p}\right) .
$$

Indeed, by plugging (A.2) into (A.1), we have

$\forall i \neq j, d_{i j, \text { clean }}^{2}\left(\mathrm{O}_{i j, \text { noisy }}^{*}\right)+\operatorname{trace}\left(\Sigma_{i}+\Sigma_{j}\right) \leq d_{i j, \text { clean }}^{2}\left(\mathrm{O}_{i j, \text { clean }}^{*}\right)+\operatorname{trace}\left(\Sigma_{i}+\Sigma_{j}\right)+\mathrm{O}_{P}\left(u_{n, p}\right)$. 
Now, by definition of $d_{i j, \text { clean }}^{2}$, we have

$$
d_{i j \text {,clean }}^{2}\left(\mathrm{O}_{i j, \text { clean }}^{*}\right) \leq d_{i j, \text { clean }}^{2}\left(\mathrm{O}_{i j, \text { noisy }}^{*}\right) .
$$

So by (A.3) and (A.4), we have shown that

$$
\begin{aligned}
\forall i \neq j, d_{i j, \text { clean }}^{2}\left(\mathrm{O}_{i j, \text { clean }}^{*}\right) & +\operatorname{trace}\left(\Sigma_{i}+\Sigma_{j}\right) \leq d_{i j, \text { clean }}^{2}\left(\mathrm{O}_{i j, \text { noisy }}^{*}\right)+\operatorname{trace}\left(\Sigma_{i}+\Sigma_{j}\right), \\
& \leq d_{i j, \text { clean }}^{2}\left(\mathrm{O}_{i j, \text { clean }}^{*}\right)+\operatorname{trace}\left(\Sigma_{i}+\Sigma_{j}\right)+\mathrm{O}_{P}\left(u_{n, p}\right) .
\end{aligned}
$$

This clearly implies that

$$
\forall i \neq j, d_{i j, \text { clean }}^{2}\left(\mathrm{O}_{i j, \text { clean }}^{*}\right) \leq d_{i j, \text { clean }}^{2}\left(\mathrm{O}_{i j, \text { noisy }}^{*}\right) \leq d_{i j, \text { clean }}^{2}\left(\mathrm{O}_{i j, \text { clean }}^{*}\right)+\mathrm{O}_{P}\left(u_{n, p}\right) .
$$

Since $u_{n, p} \rightarrow 0$ as $n$ and $p$ grow, this means that, for any given $\eta$, with $0<\eta<1$, with very high probability,

$$
\forall 1 \leq i \neq j \leq n, 0_{i j, \text { noisy }}^{*} \in \mathcal{T}_{i j, u_{n, p}^{1-\eta}}^{(k)} .
$$

We conclude, using Assumption A0, that with very high-probability,

$$
\forall 1 \leq i \neq j \leq n, d\left(0_{i j, \text { noisy }}^{*}, 0_{i j, \text { clean }}^{*}\right) \leq g\left(u_{n, p}^{1-\eta}\right) .
$$

\section{A-3. Proof of Lemma 3.2 in [21].}

Proof. Recall also that the computation of the $D$ matrix does not involve the diagonal weights. Therefore,

$$
L(W, G)=L_{0}(W, G)+D^{-1} \Delta\left(\left\{w_{i, i}\right\}, G_{i, i}\right),
$$

where $\Delta\left(\left\{w_{i, i}\right\}, G_{i, i}\right)$ is the block diagonal matrix with $(i, i)$ block diagonal $w_{i, i} G_{i, i}$, and $D^{-1} \Delta\left(\left\{w_{i, i}\right\}, G_{i, i}\right)$ is a block diagonal matrix with $(i, i)$ block diagonal

$$
\frac{w_{i, i}}{\sum_{j \neq i} w_{i, j}} G_{i, i}
$$

This implies that

$$
\left\|\mid L(W, G)-L_{0}(W, G)\right\|_{2} \leq \sup _{i} \frac{w_{i, i}}{\sum_{j \neq i} w_{i, j}}\left\|G_{i, i}\right\|_{2} .
$$

Our assumptions imply that $\sum_{j \neq i} w_{i, j}>\gamma n, \sup _{i} w_{i, i} \leq C$ and $\left\|\mid G_{i, i}\right\|_{2} \leq$ $\left\|G_{i, i}\right\|_{F} \leq C$. Hence,

$$
\left\||| L(W, G)-L_{0}(W, G)\right\|_{2} \leq \frac{C^{2}}{n \gamma} .
$$


We still have $L\left(\widetilde{W_{f}}, \widetilde{G}\right)=L(\widetilde{W}, \widetilde{G})$ and of course

$$
L_{0}\left(\widetilde{W_{f}}, \widetilde{G}\right)=L_{0}(\widetilde{W}, \widetilde{G}) .
$$

Note further that the assumptions of Lemma 3.1 in [21] apply to the matrices $\left(W \circ 1_{i \neq j}, G\right)$ and $\left(\widetilde{W} \circ 1_{i \neq j}, \widetilde{G}\right)$. Indeed, the off-diagonal conditions on the weights are the assumptions we are working under. The diagonal conditions on the weights in Lemma 3.1 in [21] are trivially satisfied here since both $W \circ 1_{i \neq j}$ and $\widetilde{W} \circ 1_{i \neq j}$ have diagonal entries equal to 0 . Hence, Lemma 3.1 in [21] gives

$$
\|\left|L_{0}(W, G)-L_{0}(\widetilde{W}, \widetilde{G})\right|_{2} \leq \frac{1}{\gamma} C(\eta+\epsilon)+\frac{\epsilon}{\gamma(\gamma-\epsilon)} C^{2} .
$$

Using Weyl's inequality (see [10]) and our bound on $\left\|\left|L(W, G)-L_{0}(W, G)\right|\right\|_{2}$, we therefore get

$$
\left\||| L(W, G)-L_{0}(\widetilde{W}, \widetilde{G})\right\|_{2} \leq \frac{1}{\gamma} C(\eta+\epsilon)+\frac{\epsilon}{\gamma(\gamma-\epsilon)} C^{2}+\frac{C^{2}}{n \gamma} .
$$

A-3.1. Extension of Lemma 3.2 in [21]. We present in this subsubsection an extension of Lemma 3.2 in [21] that removes the assumption of uniform approximation of $G_{i, j}$ by $\widetilde{G}_{i, j}$ when $L_{0}(W, G)$ can be approximated by a sparser matrix.

In what follows, $\otimes$ denotes the Kronecker product.

COROllary A-1. We work under the same setup as in Lemma 3.1 in [21] and with the same notations. We assume that there exists a $n \times n$ matrix $\mathrm{M}$ with entries in $\{0,1\}$ such that

$$
\exists\left\{f_{i}\right\}_{i=1}^{n}, f_{i}>0: \sup _{i \neq j}\left|\frac{\tilde{w}_{i, j}}{f_{i}}-w_{i, j}\right| \leq \epsilon, \text { and } \sup _{i, j: \mathrm{M}_{i, j}=1}\left\|\widetilde{G}_{i, j}-G_{i, j}\right\|_{F} \leq \eta .
$$

Suppose furthermore that there exists $C>0$ such that $0 \leq w_{i, j} \leq C$, $\sup _{i, j}\left\|G_{i, j}\right\|_{F} \leq C$, and $\sup _{i, j}\left\|\widetilde{G}_{i, j}\right\|_{F} \leq C$.

We call $M=\mathrm{M} \otimes 1_{d \times d}$, and

$$
A_{M}=L_{0}(W, G) \circ M, \text { and } \widetilde{A}_{M}=L_{0}(\widetilde{W}, \widetilde{G}) \circ M
$$

Then, if $\inf _{i} \sum_{j \neq i} w_{i, j} / n>\gamma$ and $\gamma>\epsilon$, we have

$$
\left\||| A_{M}-\tilde{A}_{M}\right\|_{2} \leq \frac{1}{\gamma} C(\eta+\epsilon)+\frac{\epsilon}{\gamma(\gamma-\epsilon)} C^{2} .
$$


Proof. The proof follows from the simple observation that

$$
A_{M}=L_{0}(W, G \circ M) \text {, and } \widetilde{A}_{M}=L_{0}(\widetilde{W}, \widetilde{G} \circ M) .
$$

The conditions of Lemma 3.2 in [21] apply in this case and immediately give us the result.

We will need in what follows the following simple lemma.

Lemma A-1. Suppose $N$ is a nd $\times$ nd block matrix with blocks of size $d \times d$. Suppose

$$
N_{i, j}=b_{i, j} G_{i, j}
$$

where $b_{i, j} \geq 0$ and $\left\|\left|G_{i, j}\right|\right\|_{2} \leq C$ where $G_{i, j}$ is a $d \times d$ matrix. Then

$$
\operatorname{trace}\left(\left(N N^{*}\right)^{k}\right) \leq \operatorname{dtrace}\left(\left(B B^{*}\right)^{k}\right) C^{2 k},
$$

and therefore

$$
\|\| N \mid\left\|_{2} \leq\right\| B\|\|_{2} C
$$

Proof. The proof is classical and relies simply of the fact that

$$
\operatorname{trace}\left(\left(N N^{*}\right)^{k}\right)=\sum_{1 \leq i_{1} \leq n} \prod_{1 \leq i_{2}, \ldots, i_{2 k} \leq n} B_{i_{1}, i_{2}} B_{i_{2}, i_{3}}^{*} \ldots B_{i_{2 k}, i_{1}}^{*} \operatorname{trace}\left(G_{i_{1}, i_{2}} G_{i_{2}, i_{3}}^{*} \ldots G_{i_{2 k, i_{1}}}^{*}\right) \text {. }
$$

Now, when $A$ and $D$ are $d$-dimensional matrices, $|\operatorname{trace}(A D)| \leq d\left|\|A\|\left\|_{2}|||D|\right\|\right|_{2}$, according to von Neumann's trace theorem (see [25], p. 183). So we conclude, using the fact that $B$ has only non-negative entries, bounding the previous sum term by term, and using the fact that $B$ is symmetric,

$$
\operatorname{trace}\left(\left(N N^{*}\right)^{k}\right) \leq d \operatorname{trace}\left(\left(B B^{*}\right)^{2 k}\right) C^{2 k} .
$$

This gives the first inequality. The second inequality comes simply from the fact that $\||N|\|_{2}=\lim _{k \rightarrow \infty}\left[\operatorname{trace}\left(\left(N^{*} N\right)^{k}\right)\right]^{1 /(2 k)}$.

We are now in position to state a generalization of Lemma 3.2 in [21] that applies even when we cannot uniformly approximate $G_{i, j}$ by $\widetilde{G}_{i, j}$. We call $L_{0}(W)=L_{0}(W, 1)$, i.e the Laplacian matrix where $G_{i, j}$ is replaced by 1 .

Lemma A-2. Suppose in the notation of Corollary A-1, that there exists a matrix $\mathrm{M}$ such that

$$
\left\|\mid L_{0}(W)-L_{0}(W) \circ \mathrm{M}\right\| \|_{2} \leq \delta
$$


Suppose that the assumptions of Corollary A-1 hold. Then we have, with $\mathrm{b}=\frac{1}{\gamma} C(\eta+\epsilon)+\frac{\epsilon}{\gamma(\gamma-\epsilon)} C^{2}$,

$$
||\left|L_{0}(W, G)-L_{0}(\widetilde{W}, \widetilde{G})\right| \|_{2} \leq 2(\mathrm{~b}+\delta) C+\mathrm{b} .
$$

Intuitively, this lemma means that for the indices $(i, j)$ such that $w_{i, j}$ 's are comparatively very small (in the sense that they do not affect much $L_{0}(W)$ spectrally), the fact that we may not be able to approximate very well $G_{i, j}$ by $\widetilde{G}_{i, j}$ for those indices is not a problem: spectrally, $L_{0}(W, G)$ and $L_{0}(\widetilde{W}, \widetilde{G})$ are still close. Hence, in certain situations, we are able to relax slightly the assumptions of Lemma 3.2 in [21], and not require a uniformly good approximation of $G_{i, j}$ by $\widetilde{G}_{i, j}$.

Proof. We first note that under our assumptions, the previous lemma implies that

$$
\left\|\left|L_{0}(W, G)-L_{0}(W, G) \circ M\right|\right\|_{2} \leq \delta C .
$$

This is a consequence of the fact that $\left(L_{0}(W, G)-L_{0}(W, G) \circ M\right)_{i, j}=b_{i, j} G_{i, j}$, with $B=L_{0}(W)-L_{0}(W) \circ \mathrm{M}$. We have already seen in Corollary A-1 that

$$
\left\|\left|L_{0}(W, G) \circ M-L_{0}(\widetilde{W}, \widetilde{G}) \circ M\right|\right\|_{2} \leq \mathrm{b} .
$$

Now applying Lemma 3.2 in $[21]$ to $L_{0}(W)$ and $L_{0}(\widetilde{W})$, we have

$$
\left\|\mid L_{0}(W)-L_{0}(\widetilde{W})\right\|_{2} \leq \mathrm{b}
$$

and

$$
\left\|L_{0}(W) \circ \mathrm{M}-L_{0}(\widetilde{W}) \circ \mathrm{M}\right\|_{2} \leq \mathrm{b},
$$

(Note that in this scalar case, we can take $\eta=0$ in the first bound, since $G_{i, j}=\widetilde{G}_{i, j}=1$. Also, the second bound can be deduced from the first by applying Lemma A-1, where $\mathrm{M}_{i, j}$ plays the role of $G_{i, j}$.) We conclude that

$$
\left\|\mid L_{0}(\widetilde{W})-L_{0}(\widetilde{W}) \circ \mathrm{M}\right\| \|_{2} \leq 2 \mathrm{~b}+\delta .
$$

It therefore follows from Lemma A-1 that

$$
\left\||| L_{0}(\widetilde{W}, \widetilde{G})-L_{0}(\widetilde{W}, \widetilde{G}) \circ M\right\|_{2} \leq(2 \mathrm{~b}+\delta) C .
$$

So we conclude that

$$
\left\|\mid L_{0}(W, G)-L_{0}(\widetilde{W}, \widetilde{G})\right\|_{2} \leq 2(\mathrm{~b}+\delta) C+\mathrm{b} .
$$




\section{A-4. Proof of Theorem 3.1 in [21].}

Proof. The proposition is a simple consequence of our previous results and Lemma 3.2 in [21]. Indeed, in the notation of Lemma 3.2 in [21], we call

$w_{i, j}=\left\{\begin{array}{ll}\exp \left(-d_{i j, \text { clean }}^{2} / \epsilon\right) & \text { if } i \neq j \\ 1 & \text { if } i=j\end{array}\right.$ and $\quad \tilde{w}_{i, j}=\left\{\begin{array}{ll}\exp \left(-d_{i j, \text { noisy }}^{2} / \epsilon\right) & \text { if } i \neq j \\ 1 & \text { if } i=j\end{array}\right.$.

Similarly, we call

$$
G_{i, j}=\left\{\begin{array}{ll}
\mathrm{O}_{i j, c l e a n}^{*} & \text { if } i \neq j \\
\operatorname{Id}_{d} & \text { if } i=j
\end{array} \text { and } \quad \widetilde{G}_{i, j}=\left\{\begin{array}{ll}
0_{i j, n o i s y}^{*} & \text { if } i \neq j \\
\operatorname{Id}_{d} & \text { if } i=j
\end{array} .\right.\right.
$$

Under Assumption G1, we know that, if $f_{i}=\exp (-2 \operatorname{trace}(\Sigma) / \epsilon), \sup _{i \neq j} \mid w_{i, j}-$ $\tilde{w}_{i, j} / f_{i} \mid=o_{P}(1)$. Similarly, under Assumptions G1, A0 and A1, we know, using Theorem 2.2 in [21] that

$$
\sup _{i, j} d\left(G_{i, j}, \widetilde{G}_{i, j}\right)=\mathrm{oo}_{P}(1)
$$

and therefore, since $k$, the parameter of $S O(k)$, is held fixed in our asymptotics,

$$
\sup _{i, j}\left\|G_{i, j}-\widetilde{G}_{i, j}\right\|_{F}=\mathrm{o}_{P}(1) .
$$

Since we assumed that

$$
\inf _{i} \sum_{j \neq i} \frac{\exp \left(-d_{i j, \text { clean }}^{2} / \epsilon\right)}{n} \geq \gamma>0
$$

i.e, in the notations of Lemma 3.2 in [21]

$$
\inf _{i} \frac{\sum_{j \neq i} w_{i, j}}{n} \geq \gamma>0
$$

where $\gamma$ is independent of $n$ and $p$, all the assumptions of Lemma 3.2 in [21] are satisfied when $n$ and $p$ are large enough, and we conclude that, in the notations of this lemma,

$$
\left\||| L_{0}(W, G)-L_{0}(\widetilde{W}, \widetilde{G})\right\|_{2}=\mathrm{o}_{P}(1) .
$$

Furthermore, we have $0 \leq w_{i, j}, \tilde{w}_{i, j} \leq 1,\left\|G_{i, j}\right\|_{F} \leq \sqrt{k}$ and $\left\|\widetilde{G}_{i, j}\right\|_{F} \leq \sqrt{k}$, the latter two results coming from the fact that the columns of $G_{i, j}$ and $\widetilde{G}_{i, j}$ have unit norm. So we conclude that

$$
\left\||| L(W, G)-L_{0}(\widetilde{W}, \widetilde{G})\right\|_{2}=\mathrm{o}_{P}(1) .
$$




\section{APPENDIX B: GRAPH CONNECTION LAPLACIAN AND VECTOR DIFFUSION MAPS FROM A POINT CLOUD: IMPLEMENTATION}

In this section, we discuss how to analyze a given point cloud by GCL and VDM. Take the dataset as $\mathcal{X}=\left\{x_{i}\right\}_{i=1}^{n}$ located in a metric space with metric $d$. First, we build up the affinity graph $\mathrm{G}=(\mathrm{V}, \mathrm{E})$, where

$$
\mathrm{v}:=\mathcal{X}
$$

and

$$
\mathrm{E}:=\{(i, j) ; i, j \in \mathrm{V}\} .
$$

Note that in practice, for the sake of computational efficiency, it is common to use a nearest-neighbor $(\mathrm{NN})$ scheme to build up the graph, denoted as $\mathrm{G}^{\mathrm{NN}, k}=\left\{\mathrm{V}, \mathrm{E}^{\mathrm{NN}, K}\right\}$, where

$$
\mathrm{E}^{\mathrm{NN}, K}:=\{(i, j) ; j \text { is the } \mathrm{k} \text {-th nearest neighbors of } i \text {, where } k \leq K\},
$$

and $K \in \mathbb{N}$ is provided by the user. There is another commonly encountered NN scheme used to construct the edges, which is defined by ranking the distances; that is,

$$
\mathrm{E}^{\mathrm{NN}}:=\left\{(i, j) ; d\left(x_{i}, x_{j}\right) \leq \epsilon\right\},
$$

and $\epsilon>0$ is the threshold provided by the user. In other words, a pair of vertices is connected if their distance is less than a threshold value. Since the NN scheme does not influence the following construction, we demonstrate the algorithm using G. If there is no further structure inside the dataset, we assign the weight function as

$$
w_{0}:(i, j) \in \mathrm{E} \mapsto e^{-d\left(x_{i}, x_{j}\right)^{2} / h},
$$

where $h>0$ is the bandwidth provided by the user, and the connection function is defined to be a trivial one, that is,

$$
r_{0}(i, j)=1 \text { for all }\left(x_{i}, x_{j}\right) \in \mathrm{E} .
$$

Hence, we have constructed the connection graph (G, $\left.w_{0}, r_{0}\right)$ which is essentially the same as the affinity graph. While there is a further structure inside the dataset, we may consider a different metric, which takes this structure into account and hence define a non-trivial connection function. Take the rotationally invariant distance (RID) for example. We then define the weight function as

$$
w:(i, j) \in \mathrm{E} \mapsto e^{-\left(\min _{R \in O(d)} d\left(x_{i}, R \circ x_{j}\right)\right)^{2} / h},
$$


where $h>0$ is the bandwidth, and

$$
r:(i, j) \in \mathrm{E} \mapsto \operatorname{argmin}_{R \in O(d)} d\left(x_{i}, R \circ x_{j}\right) \in O(d) .
$$

With $w$ and $r$, we have constructed the connection graph (G, $w, r)$. With the connection graph, we can build up the $n \times n$ affinity matrix $W$ by $W_{i j}=w(i, j)$, the connection matrix $G$ as a $n \times n$ block matrix with $d \times d$ blocks by $G_{i j}=r(i, j)$, and hence the CGL matrix $L(W, G)$ (see Equation (1) in [21]). We then evaluate the eigenvalues and eigenvectors of the CGL and study the data by analyzing the eigenstructure.

A good theory concerning the choice of bandwidth is still lacking. It is however common in practice to take one of the quartiles of the empirical distribution of $\left(\min _{R \in O(d)} d\left(x_{i}, R \circ x_{j}\right)\right)^{2}$.

B-1. Further details concerning the numerics in Subsection 4.1 in [21]. We provide more details about the simulation in Figures 1,2 and 3 . We call a 1 -dim manifold $\{(\cos (\theta), \sin (\theta)) ; \theta \in[0,2 \pi)\}$ with the induced metric from $\mathbb{R}^{2}$ the canonical $S^{1}$. First of all, as is discussed in Section D, if we uniformly independently and identically (i.i.d.) sample points from a one dimensional manifold $M^{1}$ embedded in $\mathbb{R}^{p}$ which is isometric to the canonical $S^{1}$, then asymptotically, we recover the Laplace-Beltrami operator of the canonical $S^{1}$ from the normalized GL. Clearly the top two non-trivial eigenfunctions of the Laplace-Beltrami operator are $\sin (\theta)$ and $\cos (\theta)$ and the eigenvalues are the same. Thus, asymptotically the embedding provided by the tDM with $m=2$ recover the topology or the original manifold $M^{1}-$ the embedding is diffeomorphic to $S^{1}$.

However, in this simulation with $M^{1}$ being the twisted bell-shaped simple and closed curve, when noise does not exist, the points are sampled nonuniformly from a circle embedded in $\mathbb{R}^{p}$, which is diffeomorphic but not isometric to the canonical $S^{1}$. It has been shown in $[15,45]$ that in this case the normalized GL approximates the Fokker-Planck operator. It is well known that the Fokker-Planck operator over a simple closed curve is actually a Sturm-Liouville operator with positive coefficients and periodic boundary conditions. In addition, although the eigenfunctions of the Fokker-Planck operator are not the sine and cosine functions, from the classical SturmLiouville theory we know that the tDM relying on the top two non-trivial eigenfunctions circles the origin exactly once; that is, viewing the embedding of each point as a complex number, the angle is monotonically increasing. Thus we know that the tDM based on the top two eigenfunctions of the normalized GL still provides an embedding of the original $M^{1}$ into $\mathbb{R}^{2}$. This explains why we are able to visualize the dataset by tDM with the top 
three non-trivial eigenvectors in Figure 1 and 2. The main reason we choose three but not two eigenfunctions is to match the common practice - to visualize dataset in $\mathbb{R}^{3}$. We mention that this result does not hold for a general manifold. For example, there is no way we can visualize a manifold with dimension higher than 3 in $\mathbb{R}^{3}$. We will discuss a bit more about this embedding issue in the end of Section D.

Finally, the choice of $t=1$ and $\delta=.2$ in our nearest-neighbor estimation problem is common in practice - there is no good theory guiding these choices at this point, as far as we know.

B-2. Mathematical details concerning the simulation in Subsection 4.2 in [21]. To focus specifically on demonstrating the influence of noise on this problem, we work with 2-dimensional images observed in polar coordinates. If an image is defined in Cartesian coordinates, then in general a numerical rotation will introduce numerical artifacts and errors since resampling or interpolation procedures are then involved to compare two rotated images. These numerical issues are alleviated if we work with polar coordinates. To further minimize these numerical artifacts, we use surrogate images as our dataset - by a surrogate image, we mean a function defined on the circle $S^{1}$, which is discretized into $p$ equally spaced points. In other words, we consider images defined in polar coordinates, where we only have one sample on the radial axis.

Now we discretize the $2 \times 2$ rotational group, $S O(2)$, which is the same as the circle $S^{1}$, into $p$ equally spaced points, that is, $\mathcal{T}^{(2)}:=\left\{e^{i 2 \pi k / p}\right\}_{k=1}^{p}$ - the sample $e^{i 2 \pi k / p} \in \mathcal{T}^{(2)}$ simply rotates vectors in $\mathbb{R}^{2}$ by an angle $2 \pi k / p$. Note that since the surrogate images are defined on $p$ equally spaced points on $S^{1}$, the rotations in $\mathcal{T}^{(2)}$ act exactly on the images without introducing any numerical error. We choose $n_{K}$ different surrogate images, denoted as $\left\{f_{i}\right\}_{i=1}^{n_{K}} \subset \mathbb{R}^{p}$. Then we randomly and independently rotate each of them by $n_{R}$ angles; that is, for all $k=1, \ldots, n_{K}$ and $l=1, \ldots, n_{R}$, we have $S_{i}:=R_{k, l} \circ f_{k}$, where $R_{k, l} \in \mathcal{T}^{(2)}, R_{k, l} \circ f_{k}$ means rotating $f_{k}$ by $R_{k, l}$ and $i=(k-1) n_{R}+l$. We assume that $\operatorname{argmin}_{R \in \mathcal{T}^{(2)}}\left\|f_{i}-R \circ f_{j}\right\|>0$, for all $i, j=1, \ldots, n_{K}$; that is, the image $f_{i}$ is not a rotated version of another one $f_{j}$. In the end we get $n=n_{K} n_{R}$ randomly rotated images $\left\{S_{i}\right\}_{i=1}^{n} \subset \mathbb{R}^{p}$. Denote by $\sigma$ the standard deviation of all pixels of all images in $\left\{f_{i}\right\}_{i=1}^{n_{K}}$. The data $\mathcal{X}=\left\{I_{i}\right\}_{i=1}^{n}$ we analyze is the clean data contaminated by the noise which is i.i.d. sampled from $Z$, that is, we have $I_{i}=S_{i}+Z_{i}$.

We now build up the connection graph by setting $\mathrm{V}:=\left\{I_{i}\right\}_{i=1}^{n}$ and $\mathrm{E}:=\left\{(i, j) ; I_{i}, I_{j} \in \mathrm{V}\right\}$; that is, we take the complete graph scheme. Next we assign the weight function as $w:(i, j) \mapsto e^{-d_{\mathrm{RID}}^{2}\left(I_{i}, I_{j}\right) / m}$, where $m$ is 
the $25 \%$ quantile of all non-zero RID distances defined on E, and the connection function as $r:(i, j) \mapsto \operatorname{argmin}_{R \in \mathcal{T}^{(2)}}\left\|I_{i}-R \circ I_{j}\right\|$. For comparison purposes, we also take the nearest neighbor scheme to construct the connection graph, denoted by $\left(\mathrm{G}^{\mathrm{NN}}, w^{\mathrm{NN}}, r^{\mathrm{NN}}\right)$, where we choose 100 nearest neighbors - as defined by the RID distance - to construct edges. When the images are noise-free, due to the connection function, we can recover $R_{k, l}$ 's up to a rotation from the top eigenvector $v_{1}$ of different GCL's built up from different connection graphs, $(\mathrm{G}, w, r)$ or $\left(\mathrm{G}^{\mathrm{NN}}, w^{\mathrm{NN}}, r^{\mathrm{NN}}\right)$, with or without removing the diagonal entries. To simplify the notation, we will use the same notation $v_{1}$ to denote the top eigenvector of the different GCL's. Precisely, the estimated rotation is built up from $v_{1}$, denoted as $v \in \mathbb{C}^{n}$, by setting $v(i)=\frac{v_{1}(i)}{\left|v_{1}(i)\right|}$ when $\left|v_{1}(i)\right|>0$ and $v(i)=1$ when $\left|v_{1}(i)\right|=0$. (In a slight departure from the descriptions given earlier in the paper, the $r_{i, j}$ 's are not $2 \times 2$ matrices here, but complex numbers describing the corresponding rotations. Hence, $v_{1}$ is in $\mathbb{C}^{n}$. If we had used $2 \times 2$ matrices, $v_{1}$ would have been in $\mathbb{R}^{2 n}$ and we could have computed the vector $v$ by using pairs of entries of $v_{1}$.)

To evaluate the performance of the estimated rotation when noise exists, we construct a complex vector $u \in \mathbb{C}^{n}$ whose $i$-th entry - where $i=(k-$ 1) $n_{R}+l, k=1, \ldots, n_{K}$ and $l=1, \ldots, n_{R}$ - is the complex form of $R_{k, l}$. We then evaluate the difference between the estimated rotation of the $l$-th object and the ground truth by observing the angle of $u(i)^{*} v(i)$. In other words, this quantity shows the discrepancy between the true rotation and the estimated rotation. To visualize this result, we plot the vector $z \in \mathbb{R}^{n}$ where $z(i)$ is the angle of the complex number $u(i)^{*} v(i)$. In Figure 4 p. 27 in [21], the resulting $z$ 's with $p=1000, n_{K}=5, n_{R}=200, \alpha=1 / 4$ and $c=6 \sigma$ are illustrated. Note that since there are 5 different images, we see a piecewise function with 5 different values when the images are clean.

B-2.1. Impact of diagonal of the affinity matrix. In Figure 4 in [21], we showed a way to visualize the quality of the performance of our estimators for the angles by which our surrogate images were rotated. In that example, it seems that keeping or removing the diagonal of the affinity matrix had little impact on the estimator. Looking more closely at the simulation, there are several reasons for this effect: first, we simply use the eigenvector associated with the largest eigenvalue of the matrix of interest. Second, we do not try to measure metric information, which depends on more eigenvalues and eigenvectors. For this second point, removing the diagonal resulted in a very slight improvement (of a few percent) in limited numerical work for nearest neighbor estimation in this case. Finally, in this simulation, the matrix D 
turns out to not be much larger than the matrix $L_{0}$ in terms of eigenvalue. So we are a bit removed from the worst case scenario that can happen when keeping the diagonal entries of the affinity matrix in the analysis. (We also note that the matrix $\mathrm{D}$ here is not close to the identity.)

In conclusion, the simulation demonstrates that there is no disadvantage to exclude the diagonal entries of the affinity matrix from the analysis in this case. We saw e.g in the nearest-neighbor estimation problem of Subsection 4.1 in [21] that sometimes it actually helps to remove these diagonal entries. Further numerical work, which is a bit tangential to the mainly theoretical efforts of this paper, is postponed to a future paper. So our recommendation of not using the diagonal entries of the affinity matrix on the basis of the fact that they are not necessary and can sometime degrade performance in the presence of noise is validated by our simulations.

\section{APPENDIX C: TECHNICAL RESULTS}

\section{C-1. On quadratic forms.}

Lemma C-1. Suppose $Z_{1}, \ldots, Z_{n}$ are random vectors in $\mathbb{R}^{p}$, with $Z_{i}=$ $\Sigma_{i}^{1 / 2} X_{i}$, where $X_{i}$ has mean 0 and covariance $\operatorname{Id}_{p}$. We further assume that for every convex 1-Lipschitz function $f$, if $m_{f\left(X_{i}\right)}$ is a median of $f\left(X_{i}\right)$, $P\left(\left|f\left(X_{i}\right)-m_{f\left(X_{i}\right)}\right|>t\right) \leq 2 \exp \left(-c_{i} t^{2}\right)$. $Z_{i}$ 's are possibly dependent. Let $\left\{Q_{i}\right\}_{i=1}^{n}$ be $p \times p$ positive definite matrices. Call $\left\|Q_{i}\right\|_{2}$ the largest eigenvalue of $Q_{i}$. Then we have

$$
\sup _{1 \leq i \leq n}\left|\sqrt{Z_{i}^{\prime} Q_{i} Z_{i}}-\mathbf{E}\left(\sqrt{Z_{i}^{\prime} Q_{i} Z_{i}}\right)\right|=\mathrm{O}_{P}\left(\sup _{i} \sqrt{\left\|Q_{i} \Sigma_{i} / c_{i}\right\|_{2}} \sqrt{\log n}\right) .
$$

This implies that, when $\sup _{i} \sqrt{\left\|\left|Q_{i} \Sigma_{i} / c_{i}\right|\right\|_{2}} \sqrt{\log n} \rightarrow 0$,

$\sup _{1 \leq i \leq n}\left|Z_{i}^{\prime} Q_{i} Z_{i}-\operatorname{trace}\left(\Sigma_{i} Q_{i}\right)\right|=\mathrm{O}_{P}\left(\sup _{i} \sqrt{\left\|\left|Q_{i} \Sigma_{i} / c_{i}\right|\right\|_{2}} \sqrt{\log n}\left[\sup _{i} \sqrt{\operatorname{trace}\left(\Sigma_{i} Q_{i}\right)} \vee 1\right]\right)$.

As explained in [29], the condition we require on $X_{i}$ is satisfied by many distributions. We refer also to [20] for many examples. Here are two examples. The Gaussian distribution in dimension $p$ satisfies the previous assumptions with $c_{i}=1 / 2$, independently of the dimension. When $X_{i}$ 's have independent coordinates supported on intervals of width at most $B_{i}, c_{i}$ is proportional to $1 / B_{i}$.

Proof. The map $X_{i} \rightarrow \sqrt{X_{i}^{\prime} \Sigma_{i}^{1 / 2} Q_{i} \Sigma_{i}^{1 / 2} X_{i}}$ is convex and $\sqrt{\left\|Q_{i} \Sigma_{i}\right\|_{2}}=$ $\sqrt{\left\|\mid \Sigma_{i}^{1 / 2} Q_{i} \Sigma_{i}^{1 / 2}\right\|_{2}}$-Lipschitz as a function of $X_{i}$. Indeed, it is a norm, which 
gives convexity. The Lipschitz-property comes from the triangle inequality. Hence, under our assumptions, since $Z_{i}=\Sigma_{i}^{1 / 2} X_{i}$, we have

$$
P\left(\left|\sqrt{Z_{i}^{\prime} Q_{i} Z_{i}}-\mathbf{E}\left(\sqrt{Z_{i}^{\prime} Q_{i} Z_{i}}\right)\right|>t\right) \leq 2 \exp \left(-c_{i} t^{2} /\left(\left\|\left|Q_{i} \Sigma_{i}\right|\right\|_{2}\right)\right) .
$$

By a simple union bound, we get

$$
\begin{aligned}
P\left(\sup _{1 \leq i \leq n}\left|\sqrt{Z_{i}^{\prime} Q_{i} Z_{i}}-\mathbf{E}\left(\sqrt{Z_{i}^{\prime} Q_{i} Z_{i}}\right)\right|>t\right) & \leq 2 \sum_{i=1}^{n} \exp \left(-c_{i} t^{2} /\left[\left\|Q_{i} \Sigma_{i} \mid\right\|_{2}\right]\right) \\
& \leq 2 n \exp \left(-t^{2} /\left(\sup _{i}\left\|Q_{i} \Sigma_{i} / c_{i}\right\| \|_{2}\right)\right)
\end{aligned}
$$

Taking $t_{K}=K \sqrt{\left.\log (n)\left|\| Q_{i} \Sigma_{i} / c_{i}\right|\right|_{2}}$, for $K$ a constant, gives the first result. The second result follows from remarking that $\left|a^{2}-b^{2}\right|=|a-b||a+b| \leq \mid a-$ $\left.b\right|^{2}+2|b||a-b|$. When $\sup _{i} \sqrt{\left\||| Q_{i} \Sigma_{i} / c_{i} \mid\right\|_{2}} \sqrt{\log n} \rightarrow 0$, this gives immediately $\sup _{1 \leq i \leq n}\left|Z_{i}^{\prime} Q_{i} Z_{i}-\left[\mathbf{E}\left(\sqrt{Z_{i}^{\prime} Q_{i} Z_{i}}\right)\right]^{2}\right|=\mathrm{O}_{P}\left(\sup _{i} \sqrt{\left\|Q_{i} \Sigma_{i} / c_{i} \mid\right\|_{2}} \sqrt{\log n} \sup _{i}\left[\sqrt{\operatorname{trace}\left(\Sigma_{i} Q_{i}\right)} \vee 1\right]\right)$,

after we notice that trace $\left(\Sigma_{i} Q_{i}\right)=\mathbf{E}\left(Z_{i}^{\prime} Q_{i} Z_{i}\right) \geq\left[\mathbf{E}\left(\sqrt{Z_{i}^{\prime} Q_{i} Z_{i}}\right)\right]^{2}$. Finally, using the variance bound in Proposition 1.9 of [29], we see that,

$$
\mathbf{E}\left(Z_{i}^{\prime} Q_{i} Z_{i}\right)-\left[\mathbf{E}\left(\sqrt{Z_{i}^{\prime} Q_{i} Z_{i}}\right)\right]^{2} \leq 2 \mid\left\|\Sigma_{i} Q_{i} / c_{i}\right\|_{2}
$$

Under our assumption that $\sup _{i} \sqrt{\||| Q_{i} \Sigma_{i} / c_{i}||_{2}} \sqrt{\log n} \rightarrow 0$, we have $\sup _{i} \sqrt{\|\left.\left|Q_{i} \Sigma_{i} / c_{i}\right|\right|_{2}} \rightarrow$ 0 and therefore

$$
\sup _{i}\left\|\mid \Sigma_{i} Q_{i} / c_{i}\right\|_{2}=\mathrm{o}\left(\sup _{i} \sqrt{\left\|\sum_{i} Q_{i} / c_{i}\right\|_{2}}\right) .
$$

This gives the second bound.

In the case of the Gaussian distribution, the previous bounds can be improved, using an observation found in [28].

Lemma C-2. Suppose $Z_{1}, \ldots, Z_{n}$ are random vectors in $\mathbb{R}^{p}$, with $Z_{i} \sim$ $\mathcal{N}\left(0, \Sigma_{i}\right) . Z_{i}$ 's are possibly dependent. Let $\left\{Q_{i}\right\}_{i=1}^{n}$ be $p \times p$ positive definite matrices. Then we have, if $S_{i}=\Sigma_{i}^{1 / 2} Q_{i} \Sigma_{i}^{1 / 2}$

$\sup _{1 \leq i \leq n}\left|Z_{i}^{\prime} Q_{i} Z_{i}-\operatorname{trace}\left(S_{i}\right)\right|=\mathrm{O}_{P}\left(\sup _{1 \leq i \leq n} \sqrt{\log (n)} \sqrt{\operatorname{trace}\left(S_{i}^{2}\right)}+\left\|\mid S_{i}\right\|_{2} \log (n)\right)$. 
Proof. By rotational invariance of the Gaussian distribution, we have

$$
W_{i} \triangleq Z_{i}^{\prime} Q_{i} Z_{i}-\operatorname{trace}\left(S_{i}\right) \stackrel{\mathcal{L}}{=} \sum_{k=1}^{p} \lambda_{k}\left(S_{i}\right)\left(X_{k}^{2}-1\right),
$$

where $X_{k}$ 's are i.i.d $\mathcal{N}(0,1)$. Using Lemma 1 , p, 1325 in [28], we see that

$$
P\left(\frac{\left|W_{i}\right|}{2}>\sqrt{\operatorname{trace}\left(S_{i}^{2}\right)} \sqrt{x}+\left\||| S_{i} \mid\right\|_{2} x\right) \leq \exp (-x) .
$$

Taking $x=K \log (n)$ in the previous inequality and a simple union bound gives the announced result.

\section{C-2. An approximation lemma.}

Lemma C-3. Suppose $W$ and $\widetilde{W}$ are $n \times n$ matrices. Suppose $G$ and $\widetilde{G}$ are $n d \times n d$ block matrices with $d \times d$ blocks. Let us call $S$ and $\widetilde{S}$ two $n d \times n d$ matrices with $(i, j)$-block entries

$$
S_{i, j}=w_{i, j} G_{i, j} \text { and } \widetilde{S}_{i, j}=\tilde{w}_{i, j} \tilde{G}_{i, j}
$$

Let us call $D$ and $\widetilde{D}$ two $n d \times$ nd block diagonal matrices with $(i, i)$-block entries

$$
D_{i, i}=\sum_{j \neq i} w_{i, j} \operatorname{Id}_{d} \text { and } \widetilde{D}_{i, i}=\sum_{j \neq i} \tilde{w}_{i, j} \operatorname{Id}_{d} .
$$

Finally, assuming that $D$ and $\widetilde{D}$ are invertible, let us call $L(W, G)=$ $D^{-1} S$ and $L(\widetilde{W}, \widetilde{G})=\widetilde{D}^{-1} \widetilde{S}$.

Suppose that

$$
\sup _{i, j}\left|\tilde{w}_{i, j}-w_{i, j}\right| \leq \epsilon, \text { and } \sup _{i, j}\left\|\widetilde{G}_{i, j}-G_{i, j}\right\|_{F} \leq \eta,
$$

where $\|\cdot\|_{F}$ denotes the Frobenius norm of a matrix. Suppose furthermore that there exists $C>0$ such that $0 \leq w_{i, j} \leq C$, and that $\sup _{i, j}\left\|G_{i, j}\right\|_{F} \leq C$ and $\sup _{i, j}\left\|\widetilde{G}_{i, j}\right\|_{F} \leq C$.

Then, if $\inf _{i} \sum_{j \neq i} w_{i, j} / n>\gamma$ and $\gamma>\epsilon$, we have

$$
\||| L(W, G)-L(\widetilde{W}, \widetilde{G})\|_{2} \leq \frac{1}{\gamma} C(\eta+\epsilon)+\frac{\epsilon}{\gamma(\gamma-\epsilon)} C^{2} .
$$


Proof. We have $L(W, G)=D^{-1} S=(D / n)^{-1}(S / n)$. If we call $d_{i, i}=$ $\sum_{j \neq i} w_{i, j}$ and $\tilde{d}_{i, i}=\sum_{j \neq i} \tilde{w}_{i, j}$, we see that

$$
\left|d_{i, i} / n-\tilde{d}_{i, i} / n\right| \leq \sup _{j \neq i}\left|w_{i, j}-\tilde{w}_{i, j}\right| .
$$

Hence,

$$
\sup _{1 \leq i \leq n}\left|d_{i, i} / n-\tilde{d}_{i, i} / n\right| \leq \sup _{1 \leq i \leq n} \sup _{j \neq i}\left|w_{i, j}-\tilde{w}_{i, j}\right| \leq \sup _{i, j}\left|w_{i, j}-\tilde{w}_{i, j}\right| \leq \epsilon .
$$

We conclude that

$$
\||| D / n-\widetilde{D} / n\|_{2} \leq \epsilon .
$$

Under our assumptions, it is clear that $\left\|\left|(D / n)^{-1}\right|\right\|_{2} \leq 1 / \gamma$. The previous display also implies that $\left\|\left|(\widetilde{D} / n)^{-1}\right|\right\|_{2} \leq 1 /(\gamma-\epsilon)$.

Furthermore, since

$$
(D / n)^{-1}-(\widetilde{D} / n)^{-1}=(D / n)^{-1}[D / n-\widetilde{D} / n](\widetilde{D} / n)^{-1},
$$

we see that

$$
\left\|(D / n)^{-1}-(\widetilde{D} / n)^{-1}\right\|_{2} \leq \frac{\epsilon}{\gamma(\gamma-\epsilon)} .
$$

Also,

$$
\|S / n-\widetilde{S} / n\|_{F}^{2} \leq \sup _{i, j}\left\|S_{i, j}-\widetilde{S}_{i, j}\right\|_{F}^{2}
$$

Naturally, since $S_{i, j}=w_{i, j} G_{i, j}$ and $\widetilde{S}_{i, j}=\widetilde{w}_{i, j} \widetilde{G}_{i, j}$

$$
\left\|S_{i, j}-\widetilde{S}_{i, j}\right\|_{F}^{2} \leq\left|w_{i, j}\right|^{2}\left\|G_{i, j}-\widetilde{G}_{i, j}\right\|_{F}^{2}+\left|w_{i, j}-\widetilde{w}_{i, j}\right|^{2}\left\|\widetilde{G}_{i, j}\right\|_{F}^{2} \leq C^{2}\left(\eta^{2}+\epsilon^{2}\right) .
$$

Hence,

$$
\|S / n-\widetilde{S} / n \mid\|_{2} \leq\|S / n-\widetilde{S} / n\|_{F} \leq C(\eta+\epsilon) .
$$

We also note that $\|\widetilde{S} / n\|_{F} \leq C^{2}$. So we can conclude that $\left\||| D^{-1} S-\widetilde{D}^{-1} \widetilde{S}\left|\left\|_{2} \leq\right\|\right|\left|D^{-1}(S-\widetilde{S})+\left(D^{-1}-\widetilde{D}^{-1}\right) \widetilde{S}\right|\right\|_{2} \leq \frac{1}{\gamma} C(\eta+\epsilon)+\frac{\epsilon}{\gamma(\gamma-\epsilon)} C^{2}$. 
C-3. Card $\{\mathcal{T}\}:$ an example when $\mathcal{T}_{\text {exact }}^{(k)} \subset S O(k)$. Naturally, when working with discretized images/objects with $p$ pixels/voxels, we need to also discretize $S O(k)$. In light of results like Proposition 2.1 in [21], one natural question we have to deal with concerns the cardinality of the discretized set of transformations, $\mathcal{T}^{(k)}$, and the corresponding set for companion matrices, $\mathcal{T}$. The following proposition answers this question.

The images/objects are discretized in polar coordinates. In other words, each point on our grid can be identified by its location on a ray emanating from the origin and reaching a point $p$ on the sphere of radius $r_{0}$ centered at the origin. The discretization of each ray does not have to be uniform. But this discretization is the same for all rays.

In the case of $S O(2)$, this simply means that we discretize the circle of radius $r_{0}$, and our points lay on the corresponding radii. In this situation, it is natural to represent each point on our grid through $(r, \theta)$. To give a concrete example, we assume that $\theta \in\left\{2 \pi \frac{k}{M}\right\}_{k=0}^{M-1}$ and $r \in\left\{\mathrm{r}_{1}, \ldots, \mathrm{r}_{\alpha}\right\}$ with $r_{\alpha}=r_{0}$. The discretization of $S O(2)$ corresponds simply to rotations by an angle $\theta_{k}$, where $\theta_{k}=2 \pi \frac{k}{M}$. These rotations clearly map our grid onto itself.

We assume that our images or objects, after having been uniformly discretized in polar coordinates, fit in a $k$-dimensional cube. Then we assume that the rotation group is properly discretized so that each rotation is exact in the sense that it commutes with discretization. In other words, the rotation does not change the pixel values - pixels are simply swapped and pixel values are not averaged or aggregated in other ways. Note that when the image or object is discretized in Cartesian coordinates, the discretization and rotation will not commute and a distortion is inevitable. We postpone the study of such a phenomenon to future work.

Proposition C.1. When $\mathcal{T}_{\text {exact }}^{(k)}$ is the discretized version of $S O(k)$ we just discussed, we have

$$
\operatorname{Card}\left\{\mathcal{T}_{\text {exact }}^{(k)}\right\}=\operatorname{Card}\left\{\mathcal{T}_{\text {exact }}\right\}=\mathrm{O}\left(p^{k-1}\right) .
$$

Furthermore, the elements of $\mathcal{T}_{\text {exact }}$ are permutation matrices. In particular, they are orthogonal matrices.

Comment : Proposition C.1 shows that in checking Assumption G1, we can assume that Card $\{\mathcal{T}\}$ is polynomial in $p$. This implies that Assumption G1 will be satisfied when $\max \left(\sigma_{p}, \sqrt{p} s_{p}^{2}\right)=\mathrm{o}\left([\log (n p)]^{-1 / 2}\right)$. Hence the conditions we will have to check on $\sigma_{p}$ and $\sqrt{p} s_{p}^{2}$ will be quite unrestrictive and we will see that this implies that GCL algorithms are robust to considerable amount of additive noise. 
Proof. Our polar-coordinate discretization amounts to discretizing a sphere of radius $r_{0}$ in $\mathbb{R}^{k}$ with $M$ points and discretizing each ray linking a point on that sphere to the origin along $\alpha$ points. We naturally have the relationship $M \alpha=p$.

Now elements of $\mathcal{T}_{\text {exact }}^{(k)}$ are orthogonal matrices with determinant 1 , hence they can be characterized by their action on $k-1$ vectors in $\mathbb{R}^{k}$ which span a subspace of dimension $k-1$.

Let us pick $k-1$ elements among our $M$ points on the sphere of radius $r_{0}$. We require that these $k-1$ elements span a subspace of dimension $k-1$ in $\mathbb{R}^{k}$. We call the corresponding vectors $v_{1}, \ldots, v_{k-1}$.

Suppose now that $0 \in \mathcal{T}_{\text {exact }}^{(k)}$. Then, for each $i, 0 v_{i}$ has to be one of the elements of our discretized sphere. Therefore,

$$
\operatorname{Card}\left\{\mathcal{T}_{\text {exact }}^{(k)}\right\} \leq M^{k-1}=\left(\frac{p}{\alpha}\right)^{k-1} \leq p^{k-1} .
$$

Now let $0 \in \mathcal{T}_{\text {exact }}^{(k)}$ and let $O$ be the companion matrix of 0 . Note that if $I_{i}$ is our image and $I_{i}^{\vee} \in \mathbb{R}^{p}$ is its discretized version, $O I_{i}^{\vee}$ swaps the position of the entries of the vector $I_{i}^{\vee}$, since 0 maps our grid onto itself. Hence $O$ is a permutation matrix and it is therefore orthogonal.

Another approach. We note that another approach can be employed to generate a sampling grid $\mathfrak{X}$ and an associated set of exact transformations for $k \geq 3$. Take $m$ points in $R^{k}$ denoted as $X=\left\{x_{i}\right\}_{i=1}^{m}$. Take a finite subgroup, $T$ of $S O(k)$. Now consider the sampling grid $\mathfrak{X} \triangleq\left\{R x_{i} ; R \in T, x_{i} \in X\right\}$. Since $T$ is a subgroup, we know that the sampling grid $\mathfrak{X}$ is of finite size; that is, $|\mathfrak{X}| \leq m|T|$. It is also clear that $T$ is an exact set of transforms for $\mathfrak{X}$, by simply using the fact that $T$ is a group.

Note that the standard polar coordinate grid in $\mathbb{R}^{2}$ described above can be viewed as an instance of the method we just discussed, with $T$ consisting of powers of the rotation by the angle $\frac{2 \pi}{M}$.

Note however that the classification of finite subgroups of $S O(k)$, for $k \geq 3$, imposes strong constraints on the sampling grids obtained by such a construction.

\section{APPENDIX D: BACKGROUND ON GCL METHODS}

In this section, we discuss the noise-free graph connection Laplacian (GCL) $L(W, G)$ defined in (1 in [21]), understand its asymptotical behavior under the assumption that the point clouds we collect are distributed on a manifold, and show that the GCL matrix built up under this assumption enjoy a sparsity property which allows the robustness result shown in 
this paper. In addition, we will discuss the fact that the GCL matrix can be viewed as a generalization of the graph Laplacian (GL) [45]. We will see that although GL and GCL share several similar properties but are fundamentally different. We would assume the background knowledge of differential geometry in the following discussion. For a reader who is not familiar with the subject, we refer him to $[19,23,11,7,9]$ for the topics we will encounter.

We start from some notations. Denote $\mathrm{M}$ to be a $d$-dimensional compact, connected and smooth Riemannian manifold embedded in $\mathbb{R}^{p}$ via $\iota$, where $d \leq p$. Denote the tangent bundle as $T \mathrm{M}$. The tangent plane at $y \in \mathrm{M}$ is denoted as $T_{y} \mathrm{M}$. Introduce the metric $g$ on $\mathrm{M}$ induced from the canonical metric of the ambient space $\mathbb{R}^{p}$. Denote $d\left(y, y^{\prime}\right)$ to be the geodesic distance between $y, y^{\prime} \in \mathrm{M}$. Denote by $\nabla$ the covariant derivative of the vector field, $\Delta_{g}$ the Laplace-Beltrami operator, $\nabla^{2}$ the connection Laplacian of the tangent bundle associated with the Levi-Civita connection, and by Ric the Ricci curvature of $(\mathrm{M}, g)$. We denote the spectrum of $\nabla^{2}$ (resp. $\left.\Delta_{g}\right)$ by $\left\{-\lambda_{l}\right\}_{l=0}^{\infty}$ (resp. $\left\{-\gamma_{l}\right\}_{l=0}^{\infty}$ ), where $0=\lambda_{0} \leq \lambda_{1} \leq \ldots$ (resp. $\left.0=\gamma_{0}<\gamma_{1} \leq \ldots\right)$, and the corresponding eigenspaces by $F_{l}:=\left\{X \in L^{2}(T \mathrm{M}): \nabla^{2} X=-\lambda_{l} X\right\}$ (resp. $\left.E_{l}:=\left\{\phi \in L^{2}(\mathrm{M}): \Delta_{g} \phi=-\gamma_{l} \phi\right\}\right), l=0,1, \ldots$ In general, while $\gamma_{0}=0$ always exists, $\lambda_{0}$ may not: a simple example is found considering $S^{2}$ with the standard metric. It is well known [23] that $\operatorname{dim}\left(F_{l}\right)<\infty$, the eigen-vectorfields are smooth and form a basis for $L^{2}(T \mathrm{M})$ (resp. $\operatorname{dim}\left(E_{l}\right)<\infty$, the eigenfunctions are smooth and form a basis for $L^{2}(\mathrm{M})$ ), that is, $L^{2}(T \mathrm{M})=$ $\overline{\oplus_{l \in \mathbb{N} \cup\{0\}} F_{l}}$ (resp. $L^{2}(\mathrm{M})=\overline{\oplus_{l \in \mathbb{N} \cup\{0\}} E_{l}}$ ), the completion of $\oplus_{l \in \mathbb{N} \cup\{0\}} F_{l}$ with relative to the measure induced by $g$. To simplify the statement, we assume that $\lambda_{l}$ (resp. $\gamma_{l}$ ) for each $l$ are simple and $X_{l}$ (resp. $\phi_{l}$ ) is a normalized basis of $F_{l}$ (resp. $\left.E_{l}\right)$. Denote $\mathcal{B}\left(F_{k}\right)$ (resp. $\mathcal{B}\left(E_{k}\right)$ ) the set of bases of $F_{k}$ (resp. $\left.E_{k}\right)$, which is identical to the orthogonal group $O\left(\operatorname{dim}\left(F_{k}\right)\right.$ ) (resp. $\left.O\left(\operatorname{dim}\left(E_{k}\right)\right)\right)$. Denote the set of the corresponding orthonormal bases of $L^{2}(T \mathrm{M})$ by $\mathcal{B}(T \mathrm{M}, g)=\Pi_{k=1}^{\infty} \mathcal{B}\left(F_{k}\right)\left(\operatorname{resp} . \mathcal{B}(\mathrm{M}, g)=\Pi_{k=1}^{\infty} \mathcal{B}\left(E_{k}\right)\right)$.

Given the collected data $\mathcal{X}=\left\{x_{i}\right\}_{i=1}^{n} \subset \mathbb{R}^{p}$, where $x_{i}$ are signal random vector i.i.d. sampled from a random vector $\mathrm{X}$. We assume a manifold structure inside the signal random vector; that is, we view $\mathrm{X}: \Omega \rightarrow \mathbb{R}^{p}$ as a measurable function with respect to the probability space $(\Omega, \mathcal{F}, P)$, and assume that its range is $\iota(\mathrm{M})$. Note that we cannot define the probability density function (p.d.f.) of $\mathrm{X}$ on the ambient space $\mathbb{R}^{p}$ when $d<p$ since $\iota(\mathrm{M})$ is degenerate, but we can still discuss how we sample points from $\iota(\mathrm{M})$, which leads to the p.d.f. of $\mathrm{x}$ on $M$. Indeed, we employ the following definition which is based on the induced measure [12, Section 4]. Denote $\tilde{\mathcal{B}}$ to be the Borel sigma algebra on $\iota(\mathrm{M})$, and $\tilde{P}_{\mathrm{X}}$ the probability measure of $\mathrm{X}$, defined on $\tilde{\mathcal{B}}$, induced from $P$. Assume that $\tilde{P}_{\mathrm{X}}$ is absolutely continu- 
ous with respect to the volume density on $\iota(\mathrm{M})$ associated with $g$, that is, $\mathrm{d} \tilde{P}_{\mathrm{X}}(x)=f\left(\iota^{-1}(x)\right) \iota_{*} \mathrm{~d} V(x)$, where $f: \mathrm{M} \rightarrow \mathbb{R}$ and $x \in \iota(\mathrm{M})$. We interpret $f$ as the p.d.f. of $\mathrm{X}$ on $M$. To alleviate the notation, in the following we abuse the notation and will not distinguish between $\iota(\mathrm{M})$ and $\mathrm{M}$.

Pick up the kernel function $K(x)=e^{-x^{2}}$. Note that the kernel function can be more general, for example, $K \in C^{2}(\mathbb{R})$, positive and monotonic decreasing decaying fast enough, but we focus ourselves on the Gaussian kernel to make the explanation clear. Denote $\mu_{l}^{(k)}:=\int_{\mathbb{R}^{d}}\|x\|^{l} K^{(k)}(\|x\|) \mathrm{d} x$, where $k=0,1,2, l \in \mathbb{N} \cup\{0\}$, and $K^{(k)}$ means the $k$-th order derivative of $K$. We assume $\mu_{0}^{(0)}=1$ and $\sqrt{h}$ is small enough so that $\sqrt{h}$ is smaller than the reach [35] and the injectivity radius [19] of the manifold $M, \operatorname{inj}(M)$.

D-1. Connection Graph and Affinity Graph. An affinity graph, denoted as $(\mathrm{G}, w)$, is actually a special case of the connection graph in the sense that the connection function is not defined on the affinity graph. If we take a constant function $r_{0}: \mathrm{E} \rightarrow 1$, the affinity graph becomes a connection graph $\left(\mathrm{G}, w, r_{0}\right)$.

We mention that in practice, if we decide to construct the connection graph from a given dataset, there are several different ways depending on the application and goal. For example, in addition to the examples discussed in the main context, in the geometric approach to the signal processing $[42,48]$, the affinity is defined to be the Mahalanobis distance reflecting the intrinsic property of the underlying state space; in the chair synchronization problem [26], the affinity between two chair meshes is defined based on their Hausdorff distance. The quality of the chosen affinity might influence the analysis result directly.

D-2. Connection Graph Laplacian and its Applications. Now we discuss the GCL. Consider the symmetric matrix $L_{s}(W, G):=D^{-1 / 2} S D^{-1 / 2}$, which is similar to $L(W, G)$. Since $L_{s}(W, G)$ is symmetric, it has a complete set of eigenvectors $v_{n, i}, i=1, \ldots, n d$ and its associated eigenvalues $\mu_{n, i}$, where the eigenvalues are bounded by 1 [43]; that is,

$$
L_{s}(W, G) v_{n, i}=\mu_{n, i} v_{n, i} \text {. }
$$

We would order the eigenvalues in the decreasing order. Note that the eigenvectors of $L_{s}(W, G)$ is related to those of $L(W, G)$ via $D^{-1 / 2}$.

First, note that $L(W, G)$ is an operator acting on $\boldsymbol{v} \in \mathbb{R}^{n d}$ by

$$
(L(W, G) \boldsymbol{v})[i]=\frac{\sum_{j:(i, j) \in E} w(i, j) r(i, j) \boldsymbol{v}[j]}{\sum_{k:(i, k) \in E} w(i, k)},
$$


where $\boldsymbol{v}$ can be viewed as a vector-valued function defined on $\mathrm{V}$ so that $\boldsymbol{v}[j]:=(\boldsymbol{v}((j-1) d+1), \ldots, \boldsymbol{v}(j d)) \in \mathbb{R}^{d}$. We could interpret this formula as a generalized random walk on the affinity graph. Indeed, if we view the vector-valued function $\boldsymbol{v}$ as the status of a particle defined on the vertices, when we move from one vertex to the other one, the status is modified according to the relationship between vertices encoded in $r$. We mention that depending on the connection function, the structure $L(W, G)$ might be very different, which leads to different analysis results and conclusion. We will give a precise example regarding this statement later. Now we discuss an important property of the GCL - the synchronization, which has been studied in $[14,2]$ and applied to the following problems, for example,

1. a new imaging technique aiming to obtain the atomic scale resolution images of a macro-scale object called "ptychographic imaging problem" [31];

2. a frame design called "polarization" for the phase retrieval problem $[1]$;

3. a spectral relaxation approach to solve the least squares solution of the rotational synchronization problem [50];

4. graph realization problem by synchronization over the euclidean group $[17,18]$.

Here we give an intuition about this synchronization notion. Suppose there exists a vector-valued status $\boldsymbol{v} \in \mathbb{R}^{n d}$ of norm 1 which is "synchronized" according to the encoded relationship $r$ in the sense that $\boldsymbol{v}[j]=r(j, i) \boldsymbol{v}[i]$ for all $(i, j) \in \mathrm{E}$, then $L(W, G) \boldsymbol{v}[i]$ will be the same as $\boldsymbol{v}[i]$, and hence the functional associated with the eigenvalue problem

$$
\max _{\boldsymbol{v} \in \mathbb{R}^{n d} ;\|\boldsymbol{v}\|=1} \boldsymbol{v}^{T} L(W, G) \boldsymbol{v}
$$

is maximized with the eigenvalue 1 , and its eigenvector is the synchronized vector $\boldsymbol{v}$. Thus, the top eigenvector of $L(W, G)$, when viewed as the vectorvalued status on the vertex, is the "synchronized" status with respect to the connection function. We mention that the existence of the synchronized vector-valued function is equivalent to the notion of "consistency" studied in [14].

When the connection function is constant; that is, the connection matrix $G_{0}:=\mathbf{1 1}^{T}$, where $\mathbf{1}$ is a $n \times 1$ vector with all entries 1 , the GL is defined as Id $-L\left(W, G_{0}\right)$. Notice a natural interpretation of $L\left(W, G_{0}\right)$ - since the sum of each row of $L\left(W, G_{0}\right)$ is $1, L\left(W, G_{0}\right)$ is the transition matrix associated with a random walk on $\mathrm{G}$. To avoid confusion, the eigenvectors and eigenvalues 
of $L_{s}\left(W, G_{0}\right)$ are denoted as $u_{n, i}$ and $\nu_{n, i}$,

$$
L_{s}\left(W, G_{0}\right) u_{n, i}=\nu_{n, i} u_{n, i}
$$

where $i=1, \ldots, n$, and $0 \leq \nu_{n, i} \leq 1$ are ordered in the decreasing order. As a special case of GCL, the GL has several applications which deserves discussion, for example

1. in the spectral clustering algorithm, we only need to find the first $k$ trivial eigenvectors [49, 30];

2. to evaluate the Cheeger ratio, we need the second eigenvalue [13];

3. to visualize the high dimensional data, we need the first 3 non-trivial eigenvectors;

4. in the cryo-EM problem, if we want to reconstruct the rotational position of each projection image, we need the first 9 non-trivial eigenvectors [22];

5 . in the $2 \mathrm{~d}$ random tomography problem [44], only the first 2 non-trivial eigenvectors are needed;

6. in the orientability detection problem [43], we need the first eigenvector.

More algorithms depending on the eigenstructure of the GL can be found, to mention but a few, in $[16,47,27,46,36,40,39,32]$. We comment that if the p.d.f $f$ is not uniform, then a specific normalization stated in [15] allows us to study the dynamics of the underlying dynamical system [33, 34].

D-3. Asymptotical behavior of GCL. To better understand the GCL, we focus on the frame bundle and its associated tangent bundle [43] here to simplify the exploration. For GCL associated with a more general principal bundle structure, we refer the reader to [45].

Assumption D.1. $\quad M$ is a smooth and closed d-dim manifold smoothly embedded in $\mathbb{R}^{p}$ via $\iota$. The p.d.f. $f$ is a constant function defined on $M$.

When the boundary $\partial \mathrm{M}$ is not empty or $f$ is non-uniform, its theoretical results can be found in $[15,45]$. Under Assumption D.1, we collect the data $\mathcal{X}$ independently and identically sampled from the random vector with range supported on $\iota(\mathrm{M})$ with the p.d.f. $f$ and build up the following graph. First define a graph $\mathrm{G}_{\mathrm{M}}:=(\mathrm{V}, \mathrm{E})$ by taking $\mathrm{V}=\mathcal{X}$, and $\mathrm{E}=\left\{\left(x_{i}, x_{j}\right) ; x_{i} \in \mathcal{X}\right\}$. Then define the affinity function $w$ on $\mathrm{E}$ by

$$
w:(i, j) \mapsto=e^{-\left\|x_{i}-x_{j}\right\|_{\mathbb{R} p}^{2} / h},
$$


where $h>0$ is the chosen bandwidth. Note that we choose to use the Euclidean distance, instead of the geodesic distance, to build up $w$ since in practice we have only an access to the Euclidean distance (or other metric, depending on the application). Asymptotically this discrepancy will disappear.

Assumption D.2. For each point $x_{i} \in \mathcal{X}$, we also have a sample on the frame bundle $b(i) \in O(M)$ so that the $b(i)$ is the basis of the tangent space $T_{x_{i}}$ M. In particular, we are given a group-valued function $b: \mathcal{X} \rightarrow O(d)$.

With Assumption D.2, we define the connection function on $\mathrm{E}$ as

$$
r:(i, j) \mapsto b(i)^{T} P_{x_{i}, x_{j}} b(j) \in O(d)
$$

where $P_{x_{i}, x_{j}}$ presents the parallel transport of the vector field from $x_{j}$ to $x_{i}$. As a result, we have a connection graph $\left(\mathrm{G}_{\mathrm{M}}, w, r\right)$. With $\left(\mathrm{G}_{\mathrm{M}}, w, r\right)$, we build up the GCL Id $-L(W, G)$, where $W$ and $G$ are the weight matrix and connection matrix associated with $w$ and $r$. Under this framework, the GL is when we work with the trivial line bundle associated with M.

The geometrical meaning of the connection function deserves some discussions. First, note that although all tangent planes $T_{x_{i}} \mathrm{M}$ are isomorphic to $\mathbb{R}^{d}[19]$, but they are different in the sense that we cannot "compare" $T_{x_{i}} \mathrm{M}$ and $T_{x_{j}} \mathrm{M}$ directly. Precisely, it makes sense the say $u-v$ when $u, v \in \mathbb{R}^{d}$, but we can not evaluate $u_{i}-u_{j}$ when $u_{i} \in T_{x_{i}} \mathrm{M}$ and $u_{j} \in T_{x_{j}} \mathrm{M}$. To carry out the comparison between different tangent planes, we need a bit more work. Indeed, $b(i) \in O(d)$ is a basis of the tangent plane of $T_{x_{i}} \mathrm{M}$, which practical meaning is mapping $\mathbb{R}^{d}$ isomorphically to $T_{x_{i}} \mathrm{M}$. In other words, given a vector field $Y, b(i)^{T} Y\left(x_{i}\right)$ evaluates its coordinate at $x_{i}$. The parallel transport $P_{x_{i}, x_{j}}$ is a geometrical generalization of the notion "translation" in the Euclidean space - it is an isometric map mapping $T_{x_{j}} \mathrm{M}$ to $T_{x_{i}} \mathrm{M}$. As a result, $r(i, j) \in O(d)$ is an isometric map from $\mathbb{R}^{d}$ to $\mathbb{R}^{d}$, and geometrically it maps the coordinate of a vector field at $x_{j}$, that is, $\boldsymbol{v}[j]$, to the vector field at $x_{i}$, that is, $b(j) \boldsymbol{v}[j]$, then parallelly transports $b(j) \boldsymbol{v}[j]$ to $x_{i}$, and then evaluate the coordinate of $P_{x_{i}, x_{j}} b(j) \boldsymbol{v}[j]$ with related to the basis $b(i)$. We emphasize that the connection function in the connection graph associated with the frame bundle encodes not only the geometry but also the topology of the manifold. In practice, this constraint may lead to a better understanding of the underlying data structure. For example, in the cryo-EM problem, this viewpoint leads to a better angular classification result.

We now state the pointwise convergence and the spectral convergence of the $L(W, G)$. These theorems apply to the GL, while we replace the vector 
fields by the functions with the same regularity and the connection Laplacian operator by the Laplace-Beltrami operator and $b(i)=1$ (see [45] for details).

Theorem D.3 (GCL Pointwise Convergence [43, 45]). Suppose Assumption D.1 and Assumption D.2 hold and $X \in C^{4}(T M)$. For all $x_{i}$, with high probability (w.h.p.)

$$
b(i)((\operatorname{Id}-L(W, G)) \bar{X})[i]=h \frac{\mu_{2}}{2 d} \nabla^{2} X\left(x_{i}\right)+O\left(h^{2}\right)+O\left(\frac{\sqrt{\log (n)}}{n^{1 / 2} h^{d / 4-1 / 2}}\right)
$$

where $\bar{X} \in \mathbb{R}^{n d}$ and $\bar{X}[i]=b(i)^{-1} X\left(x_{i}\right)$.

To state the spectral convergence result, define an operator $T_{\mathrm{C}, h, n}: C(T \mathrm{M}) \rightarrow$ $C(T \mathrm{M})$ :

$$
T_{\mathrm{C}, h, n} X(y):=\frac{\sum_{j} e^{-\left\|y-x_{j}\right\|^{2} / h} P_{y, x_{j}} X\left(x_{j}\right)}{\sum_{l} e^{-\left\|y-x_{l}\right\|^{2} / h}},
$$

where $X \in C(T \mathrm{M})$. To simplify the discussion, we assume that the eigenvalues of the heat kernel of the connection Laplacian $e^{t \nabla^{2}}$ are simple. When there exists an eigenvalue with multiplicity greater than 2 , the theorem can be stated using the projection operators onto the eigenspaces. We mention that in the special case GL, under the manifold setup, the point convergence theorem was first established in [5] under the uniform sampling and boundary-free assumption, and then extended to a more general setup in $[24,15,41,45]$; the spectral convergence of GL was established in $[6,49]$.

Theorem D.4 (GCL Spectral Convergence [45]). Suppose Assumption D.1 and Assumption D.2 hold and fix $t>0$. Denote $\mu_{C, t, h, i}$ to be the $i$ th eigenvalue of $T_{C, h, n}^{t / h}$ with the associated eigenvector $X_{C, t, h, i}$. Also denote $\mu_{t, i}>0$ to be the $i$-th eigenvalue of $e^{t \nabla^{2}}$ with the associated eigen-vector field $X_{t, i}$. We assume that both $\mu_{C, t, h, i}$ and $\mu_{t, i}$ decrease as $i$ increases, respecting the multiplicity. Fix $i \in \mathbb{N}$. Then there exists a sequence $h_{n} \rightarrow 0$ such that

$$
\lim _{n \rightarrow \infty} \mu_{C, t, h_{n}, i}=\mu_{t, i}, \quad \text { and } \quad \lim _{n \rightarrow \infty}\left\|X_{C, t, h_{n}, i}-X_{t, i}\right\|_{L^{2}(T M)}=0
$$

in probability.

With these Theorems, we are able to discuss why different connection functions lead to different analysis results. Consider $S^{2}$ embedded in $\mathbb{R}^{3}$ with the standard metric. If we define the connection function according to the Levi-Civita connection, then the top eigenvalue of $e^{t \nabla^{2}}$ is strictly less 
than 1 due to the hairy-ball theorem [23]. In other words, asymptotically we are not able to find a synchronized vector-valued status on it. On the other hand, if we take the trivial connection function, that is, $r(i, j)=I_{2}$, then asymptotically we obtain $\Delta_{g}$ acting on two independent functions. Since the dimension of the null space of $\Delta_{g}$ is the number of the connected components of the manifold, the top eigenvalue of the GCL with the trivial connection function is 1; that is, a synchronized vector-valued status exists. See Figure 1 for the result. The main reason leading to this difference is rooted in the connection theory, and we refer the interested reader to [11].
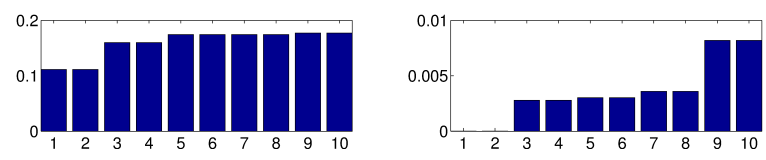

Fig 1: The first 10 eigenvalues of the $\operatorname{Id}-L(W, G)$ with related to a non-trivial connection function determined from the Levi-Civita connection of the frame bundle of $S^{2}$ (left) and a trivial connection function (right). 1000 points are sampled uniformly from $S^{2}$. Note that the eigenvalues on the right figure are the same as those of GL while the multiplicities of all the eigenvalues are 2.

D-4. The "Sparsity" of the GCL. We define the following "sparsity" condition.

Definition 1. Fix $\gamma>0$. For a $n \times n$ matrix $Q$, we sort its eigenvalues $\nu_{Q, \ell}, \ell=1, \ldots, n$, so that $\left|\nu_{Q, 1}\right| \geq\left|\nu_{Q, 2}\right| \geq \ldots \geq\left|\nu_{Q, n}\right|$. Then $Q$ satisfies the $\gamma$-sparsity property if

$$
\left|\nu_{Q, \ell}\right| \leq e^{-C_{Q} \ell^{\gamma}}
$$

for all $\ell$, where $C_{Q}>0$.

We now claim that the GCL under the manifold assumption satisfies the $2 / d$-sparsity property asymptotically.

TheOREM D.5. For $\ell \in \mathbb{N}$, asymptotically when $n \rightarrow \infty, \mu_{n, \ell} \leq e^{-C_{L} \ell^{2 / d}}$, where the constants $C_{L}>0$ depends on $d$, the lower bound of the Ricci curvature $k$ and the diameter $D$.

Proof. Note that the Weyl's theorem [9] holds for the connection Laplacian, that is,

$$
\tilde{N}(\mu) \sim \frac{1}{(4 \pi)^{d / 2} \Gamma(d / 2+1)} \mu^{d / 2},
$$


where $\tilde{N}(\mu)$ is the number of eigenvalues of $\nabla^{2}$ less than $\mu>0$, and we have the consequence that [51]

$$
\lambda_{j} \geq c^{\prime}(d, k, D) j^{2 / d}
$$

where $j \in \mathbb{N}$ and $c^{\prime}(d, k, D)$ is the universal constant depending only on $d$, the lower bound of the Ricci curvature $k$ and the diameter $D$. Note that since $\mu_{n, \ell} \rightarrow e^{-\lambda_{\ell}}$ in probability, we have

$$
\mu_{n, \ell} \leq e^{-c^{\prime}(d, k, D) \ell^{2 / d}}
$$

Hence, combined with Theorem D.4, we conclude the claim with $C_{\mathrm{L}}:=$ $c^{\prime}(d, k, D)$.

D-5. Vector Diffusion Maps and Diffusion Maps. In this subsection, we discuss a potential application of the GCL and GL - estimate the local geodesic distance. Fix $t>0$, we define the vector diffusion maps (VDM) $V_{t, n}: \mathcal{X} \rightarrow \mathbb{R}^{(n d)^{2}}$ by

$$
V_{t, n}: x_{i} \mapsto\left(\left(\mu_{n, l} \mu_{n, r}\right)^{t}\left\langle v_{n, l}[i], v_{n, r}[i]\right\rangle\right)_{l, r=1}^{n d},
$$

where $v_{n, l}[i]$ is a $d$-dim vector containing the $((i-1) d+1)$-th entry to the (id)-th entry of the eigenvector $v_{n, l}$. With this map, the Hilbert-Schmidt norm of the $(i, j)$-th block of $L_{s}(W, G)$ satisfies

$$
\left\|L_{s}(W, G)^{2 t}(i, j)\right\|_{H S}^{2}=\left\langle V_{t, n}\left(x_{i}\right), V_{t, n}\left(x_{j}\right)\right\rangle,
$$

that is, $\left\|L_{s}(W, G)^{2 t}(i, j)\right\|_{H S}^{2}$ becomes an inner product for the finite dimensional Hilbert space. The reason we need to consider $L_{s}(W, G)^{2 t}$ but not $L_{s}(W, G)^{t}$ is that all eigenvalues $\mu_{n, l}$ of $L_{s}(W, G)$ reside in the interval $[-1,1]$, and we can not guarantee the positivity of $\mu_{n, l}$ when $n$ is finite. We can then define the vector diffusion distance (VDD) to quantify the affinity between nodes $i$ and $j$ :

$$
d_{\mathrm{C}, t, n}^{2}\left(x_{i}, x_{j}\right):=\left\|V_{t, n}\left(x_{i}\right)-V_{t, n}\left(x_{j}\right)\right\|_{\ell^{2}}^{2} .
$$

In practice, we may consider the truncated vector diffusion maps ( $t V D M$ ) with diffusion time $t>0$ and accuracy $\delta>0$, which is defined as

$$
V_{t, n, m(\delta, t)}: x_{i} \mapsto\left(\left(\mu_{n, l} \mu_{n, r}\right)^{t}\left\langle v_{n, l}[i], v_{n, r}[i]\right\rangle\right)_{l, r=1}^{m(\delta, t)},
$$

where $m(\delta, t) \in \mathbb{N}$ such that $\left|\mu_{m(\delta, t)}^{t} / \mu_{2}^{t}\right|>\delta$ and $\left|\mu_{m(\delta, t)+1}^{t} / \mu_{2}^{t}\right| \leq \delta$ [43]. Similarly, we can define the truncated VDD (tVDD) as

$$
d_{\mathrm{C}, t, n, m(\delta, t)}^{2}\left(x_{i}, x_{j}\right):=\left\|V_{t, n, m(\delta, t)}\left(x_{i}\right)-V_{t, n, m(\delta, t)}\left(x_{j}\right)\right\|_{\ell^{2}}^{2} .
$$


The theoretical properties of VDM and VDD will be clear when $n \rightarrow \infty$. Fix $a \in \mathcal{B}(T \mathrm{M}, g)$ and $t>0$, define the following map embedding $x \in \mathrm{M}$ to $\ell^{2}$ :

$$
V_{t}^{a}: x \mapsto\left(\frac{(4 \pi)^{d / 2} t^{d / 2+1 / 2}}{\sqrt{d}} e^{-\left(\lambda_{k}+\lambda_{l}\right) t / 2}\left\langle X_{k}(x), X_{l}(x)\right\rangle\right)_{k, l=1}^{\infty} .
$$

With $V_{t}^{a}$, we define a new affinity between pairs of points by

$$
d_{\mathrm{C}, t}(x, y):=\left\|V_{t}^{a}(x)-V_{t}^{a}(y)\right\|_{\ell^{2}} .
$$

Due to Theorem D.3 and Theorem D.4, the VDM (resp. VDD) is a discretization of $V_{t}^{a}$ (resp. $d_{\mathrm{C}, t}$ ), so we may abuse the notation and call $V_{t}^{a}$ $\mathrm{VDM}$ and $d_{\mathrm{C}, t} \mathrm{VDD}$. We have the following Theorem saying that locally $d_{\mathrm{C}, t}$ approximates the geodesic distance:

Theorem D.6 ([43]). Take a Riemannian manifold $(M, g)$. For all $t>$ 0 , the $V D M V_{t}^{a}$ is diffeomorphic. Furthermore, suppose $x, y \in M$ so that $x=\exp _{y} v$, where $v \in T_{y} M$. When $\|v\|^{2} \ll t \ll 1$ we have

$$
d_{C, t}^{2}(x, y)=\|v\|^{2}+O\left(t\|v\|^{2}\right) .
$$

Note that if we want to estimate the geodesic distance, then we can rescale the VDM in (D.3) accordingly with the extra constant $\frac{(4 \pi)^{d / 2} t^{d / 2+1 / 2}}{\sqrt{d}}$. However, note that in practice the dimension of the manifold $d$ is unknown, and we may have to estimate it.

Although GL is a special case of GCL, with GL we may define a different embedding which has different features. Given $t>0$ and $0 \leq \delta<1$, the diffusion maps (DM) with diffusion time $t$ as

$$
\Phi_{t, n}: x_{i} \mapsto\left(\nu_{n, l}^{t} u_{n, l}(i)\right)_{l=2}^{n} .
$$

In practice, we may consider the truncated diffusion maps (tDM) with diffusion time $t>0$ and $m>0$, which is defined as

$$
\Phi_{t, n, m}: x_{i} \mapsto\left(\nu_{n, l}^{t} u_{n, l}(i)\right)_{l=2}^{m+1},
$$

where $m \in \mathbb{N}$ is chosen by the user. For the visualization purpose, we may choose $m$ to be 3 . For the other purpose, we may choose $m$ adaptively by a given threshold $\delta>0$. Indeed, $m=m(\delta, t)$ is chosen so that $\left|\lambda_{m(\delta, t)+1}^{t} / \lambda_{2}^{t}\right|>$ $\delta$ and $\left|\lambda_{m(\delta, t)+2}^{t} / \lambda_{2}^{t}\right| \leq \delta[15]$. One similar but different algorithm is the Laplacian eigenmaps $[4,5]$, that is, $x_{i}$ is mapped to $\left(u_{n, l}(i)\right)_{l=2}^{m}$, which can 
be viewed as a special DM with diffusion time $t=0$ and $1<m \leq n$ is chosen by the user. Yet another similar quantities are the global point signature proposed in [40] and the commute time embedding proposed in [38]. We mention in the Laplacian eigenmaps, global point signature and commute time embedding, the notion "diffusion" does not exist. Although these mappings are diffeomorphic to each other when $m=n$ via a linear transformation, asymptotically their behaviors are different. Furthermore, even if the connection function is trivial, the VDM and DM are different. With DM, we introduce a new metric between sampled points, which is referred to as diffusion distance (DD):

$$
d_{\mathrm{DM}, t, n}\left(x_{i}, x_{j}\right):=\left\|\Phi_{t, n}\left(x_{i}\right)-\Phi_{t, n}\left(x_{j}\right)\right\|_{\ell^{2}} .
$$

Or similarly, we may consider the truncated DD, which is defined by

$$
d_{\mathrm{DM}, t, n, m}\left(x_{i}, x_{j}\right):=\left\|\Phi_{t, n, m}\left(x_{i}\right)-\Phi_{t, n, m}\left(x_{j}\right)\right\|_{\ell^{2}} .
$$

To study $d_{\mathrm{DM}, t, n}$, we take $a \in \mathcal{B}(\mathrm{M}, g)$ and $t>0$, and map $x \in \mathrm{M}$ to the Hilbert space $\ell^{2}$ by [8]

$$
\Phi_{t}^{a}: x \mapsto\left(\sqrt{2}(4 \pi)^{d / 4} t^{d / 4+1 / 2} e^{-\gamma_{\ell} t} \phi_{\ell}(x)\right)_{\ell=1}^{\infty},
$$

With the map $\Phi_{t}^{a}$, we are able to define a new affinity between pairs of points:

$$
d_{\mathrm{DM}, t}(x, y):=\left\|\Phi_{t}^{a}(x)-\Phi_{t}^{a}(y)\right\|_{\ell^{2}} .
$$

Due to Theorem D.3 and Theorem D.4, the DM (resp. DD) is a discretization of $\Phi_{t}^{a}$ (resp. $\left.d_{\mathrm{DM}, t}\right)$, so we may abuse the notation and call $\Phi_{t}^{a} \mathrm{DM}$ and $d_{\mathrm{DM}, t}$ DD. It has been shown that the DM satisfies the following "almost isometric" property [43]:

Theorem D.7. Take a Riemannian manifold $(M, g)$. For all $t>0, \Phi_{t}^{a}$ is diffeomorphic. Furthermore, suppose $x, y \in M$ so that $x=\exp _{y} v$, where $v \in T_{y} M$. When $\|v\|^{2} \ll t \ll 1$ we have

$$
d_{\mathrm{DM}, t}^{2}(x, y)=\|v\|^{2}+O\left(t\|v\|^{2}\right) .
$$

The above theorems, when combined with the above spectral convergence theorem, says that the VDD and DD provide an accurate estimation of the geodesic between two close points. While combined with the manifold sparsity property, we have the following practical fact - if we are allowed 
a positive small error when we estimate the geodesic distance, we do not need to recover the whole eigen-structure. Instead, the first few eigenvalues and eigenvectors are enough. We have the following statement shown in $[37,3]$. Fix $\epsilon>0$ and $(\mathrm{M}, g)$ is a $d$-dim manifold satisfying $\operatorname{Ric}(g) \geq(d-$ 1) $k g, \operatorname{vol}(\mathrm{M}) \leq V, \operatorname{inj}(\mathrm{M}) \leq I$. Then there exists a $t_{0}=t_{0}(d, k, I, \epsilon)$ such that for all $0<t<t_{0}$, these is $N_{E}=N_{E}(d, k, I, V, \epsilon, t)$ so that if $N \geq N_{E}$, the truncated diffusion maps

$$
\Phi_{t, N}^{a}: x \mapsto\left(e^{-\gamma_{\ell} t} \phi_{\ell}(x)\right)_{\ell=1}^{N}
$$

is an embedding of $\mathrm{M}$ into $\mathbb{R}^{N}$ and

$$
1-\epsilon<\left|(2 t)^{(d+2) / 4} \sqrt{2}(4 \pi)^{d / 4} \mathrm{~d} \Phi_{t, N}^{a}\right| x \mid<1+\epsilon .
$$

Before ending this section, we show an interesting example regarding the data visualization and embedding issue. Take the Trefoil knot $\mathrm{M}$ embedded in $\mathbb{R}^{3}$ by $\iota(t)=[\sin (t)+2 \sin (2 t), \cos (t)-2 \cos (2 t),-\sin (3 t)]$, where $t \in[0,2 \pi)$. We refer to Figure 2 for an illustration. Note that the Trefoil knot is not homeomorphic to $S^{1}$. We sample 1000 points uniformly from $[0,2 \pi)$ independently; that is, we sample 1000 points on M non-uniformly. If we want to visualize the dataset, we may apply the tDM to embed M to $\mathbb{R}^{3}$ (or $\mathbb{R}^{2}$ ). The result is shown in Figure 2. The results deserve some discussion. Note that the tDM maps the Trefoil knot into a circle, which is not homeomorphic to the Trefoil knot; that is, the topology of the Trefoil knot is not preserved. Note that for the visualization purpose, we only choose the first 3 (or 2) eigenvectors, which leads to a map which deteriorates the topology. If we want to guarantee the preservation of the topology, we need the embedding theorem counting how many eigenvectors we need. This opens the following question, in particular when the dataset is noisy - how to balance between different data analysis results, for exampling, how to balance between preserving the topology information and data visualization?
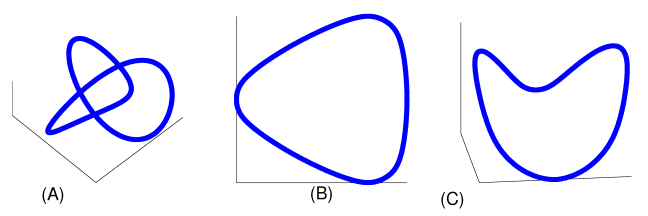

Fig 2: Left: the Trefoil knot; middle: the truncated DM with the first 2 nontrivial eigenvectors of the GL; right: the truncated DM with the first 3 non-trivial eigenvectors of the GL. 


\section{REFERENCES}

[1] B. Alexeev, A. S. Bandeira, M. Fickus, and D. G. Mixon. Phase retrieval with polarization. SIAM J. Imaging Sci., 2013.

[2] A. S. Bandeira, A. Singer, and D. A. Spielman. A Cheeger Inequality for the Graph Connection Laplacian. SIAM Journal on Matrix Analysis and Applications, 34(4):1611-1630, 2013.

[3] J. Bates. The embedding dimension of laplacian eigenfunction maps. Appl. Comput. Harmon. Anal., 2014.

[4] M. Belkin and P. Niyogi. Laplacian Eigenmaps for Dimensionality Reduction and Data Representation. Neural. Comput., 15(6):1373-1396, June 2003.

[5] M. Belkin and P. Niyogi. Towards a theoretical foundation for Laplacian-based manifold methods. In Proceedings of the 18th Conference on Learning Theory (COLT), pages 486-500, 2005.

[6] M. Belkin and P. Niyogi. Convergence of laplacian eigenmaps. In Advances in Neural Information Processing Systems 19: Proceedings of the 2006 Conference, volume 19, page 129. The MIT Press, 2007.

[7] P. Bérard. Spectral Geometry: Direct and Inverse Problems. Springer, 1986.

[8] P. Bérard, G. Besson, and S. Gallot. Embedding riemannian manifolds by their heat kernel. Geom. Funct. Anal., 4:373-398, 1994. 10.1007/BF01896401.

[9] N. Berline, E. Getzler, and M. Vergne. Heat Kernels and Dirac Operators. Springer, 2004.

[10] Rajendra Bhatia. Matrix analysis, volume 169 of Graduate Texts in Mathematics. Springer-Verlag, New York, 1997.

[11] R. L. Bishop and R. J. Crittenden. Geometry of Manifolds. Amer Mathematical Society, 2001.

[12] M.-Y. Cheng and H.-T. Wu. Local linear regression on manifolds and its geometric interpretation. J. Am. Stat. Assoc., 108:1421-1434, 2013.

[13] F. Chung. Spectral Graph Theory. American Mathematical Society, 1996.

[14] F. Chung, W. Zhao, and M. Kempton. Ranking and sparsifying a connection graph. In Anthony Bonato and Jeannette Janssen, editors, Algorithms and Models for the Web Graph, volume 7323 of Lecture Notes in Computer Science, pages 66-77. Springer Berlin Heidelberg, 2012.

[15] R. R. Coifman and S. Lafon. Diffusion maps. Appl. Comput. Harmon. Anal., 21(1):530, 2006.

[16] R. R. Coifman and M. Maggioni. Diffusion wavelets. Appl. Comput. Harmon. Anal., 21(1):53-94, 2006.

[17] M. Cucuringu, Y. Lipman, and A. Singer. Sensor network localization by eigenvector synchronization over the euclidean group. ACM Transactions on Sensor Networks, 8(3):19:1-19:42, 2012.

[18] M. Cucuringu, A. Singer, and D. Cowburn. Eigenvector synchronization, graph rigidity and the molecule problem. Information and Inference: A Journal of the IMA, $1: 21-67,2012$.

[19] M.P. do Carmo and F. Flaherty. Riemannian Geometry. Birkhauser Boston, 1992.

[20] Noureddine El Karoui. Concentration of measure and spectra of random matrices: Applications to correlation matrices, elliptical distributions and beyond. The Annals of Applied Probability, 19(6):2362-2405, December 2009. 
[21] Noureddine El Karoui and Hau-tieng Wu. Graph connection laplacian methods can be made robust to noise. page http://arxiv.org/abs/1405.6231, 2014.

[22] D. Giannakis, P. Schwander, and A. Ourmazd. The symmetries of image formation by scattering. i. theoretical framework. Opt. Express, 20(12):12799-12826, Jun 2012.

[23] P. Gilkey. The Index Theorem and the Heat Equation. Princeton, 1974.

[24] M. Hein, J. Audibert, and U. von Luxburg. From graphs to manifolds - weak and strong pointwise consistency of graph Laplacians. In Proceedings of the 18th Conference on Learning Theory (COLT), pages 470-485, 2005.

[25] Roger A. Horn and Charles R. Johnson. Topics in matrix analysis. Cambridge University Press, Cambridge, 1994. Corrected reprint of the 1991 original.

[26] Q.-X. Huang, H. Su, and L. Guibas. Fine-grained semi-supervised labeling of large shape collections. ACM Transactions on Graphics (TOG), 32(6):190, 2013.

[27] P. W. Jones, M. Maggioni, and R. Schul. Manifold parametrizations by eigenfunctions of the Laplacian and heat kernels. P. Natl. Acad. Sci. USA, 105(6):1803-8, February 2008.

[28] B. Laurent and P. Massart. Adaptive estimation of a quadratic functional by model selection. Ann. Statist., 28(5):1302-1338, 2000.

[29] M. Ledoux. The concentration of measure phenomenon, volume 89 of Mathematical Surveys and Monographs. American Mathematical Society, Providence, RI, 2001.

[30] R. Lee, J, S. Oveis Gharan, and L. Trevisan. Multi-way spectral partitioning and higher-order cheeger inequalities. In Proceedings of the Forty-fourth Annual ACM Symposium on Theory of Computing, STOC '12, pages 1117-1130, 2012.

[31] S. Marchesini, Y.-C. Tu, and H.-T. Wu. Alternating Projection, Ptychographic Imaging and Phase Synchronization. ArXiv e-prints, 2014. arXiv:1402.0550 [math.OC].

[32] F. Mémoli. A spectral notion of gromov-wasserstein distance and related methods. Appl. Comput. Harmon. Anal., 30(3):363 - 401, 2011.

[33] B. Nadler, S. Lafon, R. R. Coifman, and I. G. Kevrekidis. Diffusion maps, spectral clustering and eigenfunctions of Fokker-Planck operators. In Y. Weiss, B. Schölkopf, and J. Platt, editors, Adv. Neur. In., volume 18, pages 955-962, Cambridge, MA, 2006. MIT Press.

[34] B. Nadler, S. Lafon, R. R. Coifman, and I. G. Kevrekidis. Diffusion maps, spectral clustering and reaction coordinates of dynamical systems. Appl. Comput. Harmon. Anal., 21(1):113-127, 2006.

[35] P. Niyogi, S. Smale, and S. Weinberger. Finding the homology of submanifolds with high confidence from random samples. In Twentieth Anniversary Volume:, pages 1-23. Springer New York, 2009.

[36] M. Ovsjanikov, J. Sun, and L. Guibas. Global intrinsic symmetries of shapes. In Proceedings of the Symposium on Geometry Processing, SGP '08, pages 1341-1348. Eurographics Association, 2008.

[37] J. W. Portegies. Embeddings of riemannian manifolds with heat kernels and eigenfunctions. ArXiv e-prints, arXiv:1311.7568 [math.DG], 2013.

[38] H. Qiu and E.R. Hancock. Clustering and embedding using commute times. Pattern Analysis and Machine Intelligence, IEEE Transactions on, 29(11):1873-1890, Nov 2007.

[39] M. Reuter. Hierarchical Shape Segmentation and Registration via Topological Features of Laplace-Beltrami Eigenfunctions. Int. J. Comput. Vision, 89:287-308, 2010. 
[40] R. M. Rustamov. Laplace-beltrami eigenfunctions for deformation invariant shape representation. In Proceedings of the fifth Eurographics symposium on Geometry processing, SGP '07, pages 225-233. Eurographics Association, 2007.

[41] A. Singer. From graph to manifold Laplacian: The convergence rate. Appl. Comput. Harmon. Anal., 21(1):128-134, 2006.

[42] A. Singer and R. R. Coifman. Non-linear independent component analysis with diffusion maps. Appl. Comput. Harmon. Anal., 25(2):226 - 239, 2008.

[43] A. Singer and H.-T. Wu. Vector diffusion maps and the connection Laplacian. Comm. Pure Appl. Math., 65(8):1067-1144, 2012.

[44] A. Singer and H.-T. Wu. 2-d tomography from noisy projections taken at unknown random directions. SIAM J. Imaging Sci., 6(1):136-175, 2013.

[45] A. Singer and H.-T. Wu. Spectral convergence of the connection laplacian from random samples. submitted, 2013.

[46] J. Sun, M. Ovsjanikov, and L. Guibas. A concise and provably informative multiscale signature based on heat diffusion. In Proceedings of the Symposium on Geometry Processing, SGP '09, pages 1383-1392. Eurographics Association, 2009.

[47] A. D. Szlam, M. Maggioni, and R. R. Coifman. Regularization on Graphs with Function-adapted Diffusion Processes. The Journal of Machine Learning Research, 9:1711-1739, 2008.

[48] R. Talmon, I. Cohen, S. Gannot, and R. Coifman. Diffusion maps for signal processing: A deeper look at manifold-learning techniques based on kernels and graphs. Signal Processing Magazine, IEEE, 30(4):75-86, July 2013.

[49] U. von Luxburg, M. Belkin, and O. Bousquet. Consistency of spectral clustering. Ann. Stat., 36(2):555-586, 2008.

[50] L. Wang and A. Singer. Exact and Stable Recovery of Rotations for Robust Synchronization. Information and Inference: A Journal of the IMA, 2013. accepted for publication.

[51] H.-T. Wu. Embedding riemannian manifolds by the heat kernel of the connection laplacian. submitted, 2012.

Department of Statistics,

UC BERKELEY,

BERKELEy, CA, USA.

E-MAIL: nkaroui@berkeley.edu
Department of Mathematics,

University of TORONTO,

Toronto, Ontario, Canada.

E-MAIL: hauwu@math.toronto.edu 